\title{
Generalizations of Montessus's THEOREM ON THE ROW CONVERGENCE OF RATIONAL INTERPOLATIONS
}

\author{
Xiaoyan Liu
}

\begin{abstract}
In this paper, we investigate the convergence of the rows (with fixed denominator degrees) of the rational interpolations to a meromorphic function. We give an explicit algorithm for determining when convergence is guaranteed and obtain rates of convergence in the appropriate cases. Furthermore, we show some applications of our results to the zero distribution of orthogonal polynomials.
\end{abstract}

\section{Introduction}

Let $\Pi_{m}$ denote the collection of all algebraic polynomials of degree at most $m$. A rational function $r_{m, n}(z)$ is said to be of type $(m, n)$ if, for $p_{m} \in \Pi_{m}$ and $q_{n} \in \Pi_{n}$,

$$
r_{m, n}(z)=p_{m}(z) / q_{n}(z), \quad q_{n}(z) \not \equiv 0 .
$$

If $f(z)$ is analytic at $z=0$ (in this paper, when we say $f(z)$ is analytic at $z_{0}$, we mean that $f(z)$ is analytic in a neighborhood of $z_{0}$ ), then for each pair of non-negative integers $(n, \mu)$, there exist polynomials $P_{n, \mu}(z) \in \Pi_{n}$ and $Q_{n, \mu}(z)(\not \equiv 0) \in \Pi_{\mu}$ such that

$$
Q_{n, \mu}(z) f(z)-P_{n, \mu}(z)=O\left(z^{n+\mu+1}\right) \quad \text { as } z \rightarrow 0 .
$$

The rational function $P_{n, \mu}(z) / Q_{n, \mu}(z)$ is unique and is called the Padé approximant of type $(n, \mu)$ to $f(z)$. We denote this Padé approximant by $[n / \mu](z)$. Thus, for each $f(z)$, there corresponds a doubly-infinite array known as the Padé table. Concerning the row convergence of the Padé table, we have the following classical result of de Montessus de Ballore [4].

Theorem A. Let $f(z)$ be analytic at $z=0$ and meromorphic with precisely $\mu$ poles (counting multiplicity) in the disk $|z|<\rho$. Let $D$ denote the domain obtained from $|z|<\rho$ by deleting the $\mu$ poles of $f(z)$. Then for $n$ sufficiently large, the Padé approximant $[n / \mu](z)$ of type $(n, \mu)$ satisfies

$$
f(z)-[n / \mu](z)=O\left(z^{n+\mu+1}\right) .
$$

Each $[n / \mu](z)$, for $n$ large, has precisely $\mu$ finite poles and, as $n \rightarrow \infty$, these poles approach, respectively, the $\mu$ poles of $f(z)$ in $|z|<\rho$. The sequence $\{[n / \mu](z)\}_{n=0}^{\infty}$ converges to $f(z)$ throughout $D$, uniformly on any compact subset of $D$.

Padé approximation is the interpolation at the point $z=0$. The generalized Padé approximation is called a multipoint Padé approximation, i.e., rational interpolation. Let us have the definition first. Let

$$
\left\{\beta_{1}^{(0)} ; \beta_{1}^{(1)}, \beta_{2}^{(1)} ; \ldots ; \beta_{1}^{(n)}, \beta_{2}^{(n)}, \ldots, \beta_{n+1}^{(n)} ; \ldots\right\}
$$

Received December 5, 1994, revised May 30, 1995.

1991 Mathematics Subject Classification. 41A20, 41A25, 30E10, 41A21.

Key words and phrases: rational approximation, rational interpolation, multi-point Padé approximation, rate of convergence. 
be a triangular system of points (not necessarily distinct). Define the associated polynomials

$$
\omega_{l}(z):=\prod_{j=1}^{l+1}\left(z-\beta_{j}^{(l)}\right) \quad \text { on } \quad \Lambda(l):=\left\{\beta_{1}^{(l)}, \beta_{2}^{(l)}, \ldots, \beta_{l+1}^{(l)}\right\}, \quad l \geq 0 .
$$

If $f(z)$ is analytic in $\Lambda(n+\mu)$ for each pair of non-negative integers $(n, \mu)$, then there exist polynomials $p_{n, \mu} \in \Pi_{n}$ and $q_{n, \mu}(\not \equiv 0) \in \Pi_{\mu}$ such that

$$
\frac{f(z) q_{n, \mu}(z)-p_{n, \mu}(z)}{\omega_{n+\mu}(z)}
$$

is analytic in the set $\Lambda(n+\mu)$. We call the rational function $p_{n, \mu}(z) / q_{n, \mu}(z)$ a multipoint Padé approximant of type $(n, \mu)$. It is unique and denoted by $R_{n, \mu}(z)=$ $R_{n, \mu}(f, \Lambda(n+\mu) ; z)$. For multipoint Padé approximant, we have an analogue of Theorem A due to Saff (cf. [8]).

In this paper, we will extend Theorem $\mathrm{A}$ and its analogue to the general case where the degree of the denominator of the interpolation does not equal the number of poles of the approximated function $f(z)$. We will state our main theorem and give notation in Section 2. The proof of the main theorem will be given in Section 3. The divergence in the complement set is discussed in Section 4. For the rational interpolation rows not satisfying our criteria, we will give an example in Section 5 to explain the complexity of convergence. Furthermore, an example of an application is shown in Section 6 .

\section{Results on the multipoint Padé approximants}

Here we will study the behavior of rational interpolation on a more general point set $E$ than on a disk. We assume that $E$ is connected and equal to the closure of the interior of a finite number of mutually exterior Jordan curves $C_{1}, C_{2}, \ldots, C_{k}$. Each $C_{i}$ is of class $A$, i.e., each $C_{i}$ can be represented parametrically in terms of arc length $s$ by $x=x(s), y=y(s)$ where $x(s)$ and $y(s)$ possess second derivatives with respect to $s$ which satisfy a Lipschitz condition of some positive order in $s$. We summarize these assumptions by saying that $E$ has Property A. Furthermore, let $G(z)$ be the Green's function of $K:=\overline{\mathbf{C}} \backslash E$ having a pole at infinity, $\Delta$ be the logarithmic capacity for the point set $E$, and set

$$
\Gamma_{\rho}:=\{z ; G(z)=\log \rho\}, \quad E_{\rho}:=\operatorname{int}\left(\Gamma_{\rho}\right), \quad H(z):=-\frac{\partial G(z)}{\partial x}+i \frac{\partial G(z)}{\partial y} .
$$

Theorem 1. Suppose $E$ has property A. Let $\rho$ be a fixed positive number, $f(z)$ be analytic on $E$, meromorphic with precisely $\mu(\geq 0)$ poles (counting multiplicity) in $E_{\rho}$, and analytic on $\Gamma_{\rho}$ except for poles in the $m$ distinct points $\alpha_{1}, \alpha_{2}, \ldots, \alpha_{m}$ on $\Gamma_{\rho}$ of respective orders $r_{1}, r_{2}, \ldots, r_{m}\left(r_{1} \geq r_{2} \geq \cdots \geq r_{m}\right)$. Let the triangular schemes (1) have no limit point exterior to $E$ and satisfy the relations

$$
\begin{gathered}
\left|\omega_{n}(z)\right| \leq M \Delta^{n} \quad \text { for } z \in E \\
\left|G(z)+\log \Delta-n^{-1} \log \right| \omega_{n}(z)|| \leq M n^{-1},
\end{gathered}
$$

uniformly in $z$ on each closed bounded subset of $K$. If none of $\left\{\alpha_{i}\right\}_{i=1}^{i=m}$ is a critical point of $G(z)$, then for "good" $\nu$ (see Definition 1 below) and for all $n$ sufficiently large, there exists a unique multipoint Padé approximant $R_{n, \mu+\nu}(z):=R(f, \Lambda(n+\mu+\nu) ; z)$ of type $(n, \mu+\nu)$, which interpolates to $f(z)$ on the set $\Lambda(n+\mu+\nu)$. Each $R_{n, \mu+\nu}(z)$ has precisely $\mu+\nu$ finite poles, $\mu$ of which, as $n \rightarrow \infty$, approach the $\mu$ poles of $f(z)$ in $E_{\rho}$, respectively, and $p_{\nu, i}$ of which approach the points $\alpha_{i}$, respectively (the $\left\{p_{\nu, i}\right\}$ are 
defined in Definition 2 and satisfy $\left.\sum_{i=1}^{m} p_{\nu, i}=\nu\right)$. Let $D_{\rho}$ denote the region obtained from $E_{\rho}$ by deleting the $\mu$ poles of $f(z)$ in $E_{\rho}$, then the sequence $\left\{R_{n, \mu+\nu}(z)\right\}_{n=0}^{\infty}$ converges to $f(z)$ throughout $D_{\rho}$, uniformly on any compact subset of $D_{\rho}$, and

$$
\left\|f(z)-R_{n, \mu+\nu}(z)\right\|_{E} \leq \frac{A n^{\lambda}}{\rho^{n}}
$$

where $\lambda=\max _{1 \leq i \leq m}\left\{r_{i}-2 p_{i}-1\right\}$. Furthermore, if we let $q_{n}(z)$ denote the monic denominator of $R_{n, \mu+\nu}(z)$ and let $\pi(z)$ denote the monic polynomial of degree $\mu$ having $\mu$ poles of $f(z)$ in $E_{\rho}$ as its zeros, then

$$
\left\|q_{n}(z)-\pi(z) \prod_{i=1}^{m}\left(z-\alpha_{i}\right)^{p_{\nu, i}}\right\|_{E} \leq C / n .
$$

Before stating Definition 1, we introduce some needed notation. Here $[x]$ stands for the integer part of $x$ and an empty sum is defined to be zero. Let

$$
\begin{array}{rlrl}
u_{i, j} & :=\left[\left(r_{i}-r_{j+1}\right) / 2\right], & 1 \leq i \leq j+1 \leq m ; \\
w_{i, j}:=r_{i}-r_{j+1}-2 u_{i, j}, & 1 \leq i \leq j+1 \leq m ; \\
s_{l}:=\sum_{i=1}^{l-1} w_{i, l-1}, & 1 \leq l \leq m ; \\
s_{l}:=\sum_{i=1}^{2 m-l} w_{i, 2 m-l}, & 1+m \leq l<2 m ; \\
v_{0}:=0, & \\
v_{l}:=\sum_{i=1}^{l} u_{i, l}, & 1 \leq l \leq m-1 ; \\
v_{l}:=\sum_{i=1}^{2 m-l-1} u_{i, 2 m-l-1}+(2 m-l-1) r_{2 m-l}+\sum_{j=2 m-l}^{m} r_{j}, & m \leq l<2 m ; \\
I_{l}:=\left[v_{l-1}, v_{l}\right), & & 1 \leq l \leq m-1 ; \\
I_{m}:=\left[v_{m-1}, v_{m}\right], & & \\
I_{l}:=\left(v_{l-1}, v_{l}\right], & & m+1 \leq l<2 m .
\end{array}
$$

It is straightforward to verify that the points $v_{l}$ satisfy $v_{0} \leq v_{1} \leq \cdots \leq v_{2 m-1}$, $v_{2 m-1}=\sum_{i=1}^{m} r_{i}$, and $\left[0, v_{2 m-1}\right]=\bigcup_{l=1}^{2 m-1} I_{l}$, where the intervals are pairwise disjoint. We remark that some of the intervals $I_{l}$ may be empty.

Definition 1. For a fixed $\nu, 0 \leq \nu \leq v_{2 m-1}$, let $I_{l}, l=l(\nu)$, be the unique interval containing $\nu$. Set

$$
\begin{array}{lll}
k_{l}:=\left[\left(\nu-v_{l-1}\right) / l\right], & q_{l}:=\nu-v_{l-1}-l k_{l}, & \text { if } 1 \leq l \leq m ; \\
k_{l}:=\left[\left(\nu-v_{l-1}\right) /(2 m-l)\right], & q_{l}:=\nu-v_{l-1}-(2 m-l) k_{l}, & \text { if } m+1 \leq l \leq 2 m-1 .
\end{array}
$$

We say that $\nu$ is good if $q_{l}=0$ or $q_{l}=s_{l}$. If $0<\nu<v_{2 m-1}$ is not good, we say that $\nu$ is bad.

Next, for good $\nu$, we specify the quantities $\left\{p_{\nu, i}\right\}$ mentioned in Theorem 1 . 
Definition 2. Given a good $\nu$, we first determine the number $l$ such that $\nu \in I_{l}$; then, for this $l=l(\nu)$, we consider two cases in Definition 1 .

Case 1. $q_{l}=0$.

(a) If $1 \leq l \leq m$, then

$$
p_{\nu, i}:= \begin{cases}k_{l}+u_{i, l-1}, & 1 \leq i \leq l \\ 0, & l<i \leq m\end{cases}
$$

(b) if $m+1 \leq l \leq 2 m-1$, then

$$
p_{\nu, i}:= \begin{cases}k_{l}+u_{i, 2 m-l}+r_{2 m-l+1}, & 1 \leq i \leq 2 m-l \\ r_{i}, & 2 m-l<i \leq m .\end{cases}
$$

Case 2. $q_{l}=s_{l} \neq 0$.

(a) If $1 \leq l \leq m$, then

$$
p_{\nu, i}:= \begin{cases}k_{l}+u_{i, l-1}+w_{i, l-1}, & 1 \leq i \leq l \\ 0, & l<i \leq m\end{cases}
$$

(b) if $m+1 \leq l \leq 2 m-1$, then

$$
p_{\nu, i}:= \begin{cases}k_{l}+u_{i, 2 m-l}+r_{2 m-l+1}+w_{i, 2 m-l}, & 1 \leq i \leq 2 m-l \\ r_{i}, & 2 m-l<i \leq m .\end{cases}
$$

We have some remarks about our definitions.

Remark. We assume that $f(z)$ has $\mu$ poles in $E_{\rho}$ and $v_{2 m-1}$ poles on $\Gamma_{\rho}$ in our Theorem 1. The analogue of Theorem A guarantees that the rational interpolation rows $\left\{R_{n, \mu}(z)\right\}_{n=0}^{\infty}$ and $\left\{R_{n, \mu+v_{2 m-1}}(z)\right\}_{n=0}^{\infty}$ converge. Here, for $0<\nu<v_{2 m-1}$, we are trying to find which "intermediate" row $\left\{R_{n, \mu+\nu}(z)\right\}_{n=0}^{\infty}$ converges or which $\nu$ is good. We divide the integer interval $\left[0, v_{2 m-1}\right]$ into $2 m-1$ intervals $\left\{I_{l}\right\}$. Each nonempty interval has at least one good $\nu$. Definition 1 gives explicit criteria for whether $\nu$ is good or not. For example, $q_{1} \equiv 0$, so all $\nu \in I_{1}$ are good. The same is true for $\nu \in I_{2 m-1}$. And if $\nu$ is one of $v_{i}$ for $1 \leq i \leq m$, then $\nu$ is good. Furthermore, if $r_{1}-r_{2}$ is odd, then all $\nu \in I_{2}$ are good. In fact, it is simple to write a program to compute all good $\nu$ and to verify if a given $\nu$ is good or bad for the input $r_{1}, r_{2}, \ldots, r_{m}$.

\section{Proof of the main theorem}

To prove Theorem 1 , we need a Lemma first. We will let $L_{n+\mu+\nu}(h(z) ; z)$ denote the unique polynomial of degree $\leq n+\mu+\nu$ which interpolates $h(z)$ on the set $\Lambda(n+\mu+\nu)$.

Lemma 1. Let $E$ be the same as in Theorem 1. Let $F(z)$ be analytic on $E$, meromorphic with precisely $\mu(\geq 0)$ poles in $E_{\rho}$, and analytic on $\Gamma_{\rho}$ except for a pole at $\alpha$ of degree $r, H(\alpha) \neq 0$. Let $g_{n}(z):=L_{n+\mu+\nu}(F(z) ; z)$. Then

$$
\begin{aligned}
& \lim _{n \rightarrow \infty} n^{-(r+k)} g_{n}^{(k)}(\alpha)=\frac{(-1)^{k} H(\alpha)^{r+k} B_{r}}{(r-1) !(r+k)}, \quad k=0,1,2, \ldots, \\
& \left|F(z)-g_{n}(z)\right| \leq L(z) n^{r-1}\left|\frac{\omega_{n+\mu+\nu}(z)}{\omega_{n+\mu+\nu}(\alpha)}\right|, \quad \text { for fixed } z \neq \alpha,
\end{aligned}
$$

where $L(z)$ does not depend on $n$. 
Proof. Write

$$
F(z)=: u(z)+\sum_{i=1}^{r} B_{i}(z-\alpha)^{-i}
$$

where $u(z)$ is analytic on $\Gamma_{\rho}$. Let $s_{n, i}(z):=L_{n+\mu+\nu}\left((z-\alpha)^{-i} ; z\right), i=1,2, \ldots, r$, and $\theta_{n+\mu+\nu}(z):=1 / \omega_{n+\mu+\nu}(z)$. From the definition of $s_{n, i}(z)$, we see that

$$
(z-\alpha)^{-i}-s_{n, i}(z)=\frac{\omega_{n+\mu+\nu}(z) p_{n, i}(z)}{(z-\alpha)^{i}}
$$

where $p_{n, i}(z)$ is a polynomial of degree $i-1$. The equivalence of (11) with

$$
s_{n, i}(z)=\frac{\omega_{n+\mu+\nu}(z)\left[\theta_{n+\mu+\nu}(z)-p_{n, i}(z)\right]}{(z-\alpha)^{i}}
$$

implies (since $s_{n, i}(z)$ has no finite pole and $\alpha$ is not a zero of $\omega_{n+\mu+\nu}(z)$ ) that $p_{n, i}(z)$ interpolates $\theta_{n}(z)$ in the point $\alpha$ considered of multiplicity $i$. This property of interpolation also is possessed by the polynomial

$$
q_{n, i}(z):=\sum_{j=0}^{i-1} \theta_{n}^{(j)}(\alpha)(z-\alpha)^{j} / j !
$$

and since both $p_{n, i}(z)$ and $q_{n, i}(z)$ are of degree $i-1$, we have $p_{n, i}(z) \equiv q_{n, i}(z)$. Thus

$$
s_{n, i}(z)=\frac{\omega_{n+\mu+\nu}(z)\left(\theta_{n+\mu+\nu}(z)-\sum_{t=0}^{i-1} \theta_{n+\mu+\nu}^{(t)}(\alpha)(z-\alpha)^{t} / t !\right)}{(z-\alpha)^{i}} .
$$

From (2), we get

$$
\lim _{n \rightarrow \infty} n^{-1} \omega_{n+\mu+\nu}^{\prime}(z) \theta_{n+\mu+\nu}(z)=-H(z),
$$

uniformly on each compact subset of $K$, and by induction, we can prove (proof of Theorem 1, [7]) that

$$
\begin{gathered}
\lim _{n \rightarrow \infty} n^{-k} \omega_{n+\mu+\nu}(z) \theta_{n+\mu+\nu}^{(k)}(z)=H(z)^{k}, \\
\lim _{n \rightarrow \infty} n^{-k} \omega_{n+\mu+\nu}^{(k)}(z) \theta_{n+\mu+\nu}(z)=(-1)^{k} H(z)^{k},
\end{gathered}
$$

uniformly on each compact subset of $K$. Thus, we get

$$
\lim _{n \rightarrow \infty} n^{-(t+j)} \omega_{n+\mu+\nu}^{(t)}(\alpha) \theta_{n+\mu+\nu}^{(j)}(\alpha)=(-1)^{t} H(\alpha)^{t+j} .
$$

Furthermore, by (12), we get for $k \geq 0$ that

$$
\lim _{n \rightarrow \infty} n^{-(i+k)} s_{n, i}^{(k)}(\alpha)=\frac{(-1)^{k} H(\alpha)^{i+k}}{(i-1) !(i+k)}, \quad i=1,2, \ldots, r .
$$

Now let $s_{n, 0}(z):=L_{n+\mu+\nu}(u(z) ; z)$ and

$$
g_{n}(z)=s_{n, 0}(z)+\sum_{i=1}^{r} B_{i} s_{n, i}(z)
$$

then $g_{n}(z)$ interpolates $F(z)$ on the set $\Lambda(n+\mu+\nu)$. Using (15) for $i=0,1, \ldots, r$, one obtains

$$
\lim _{n \rightarrow \infty} n^{-(r+k)} g_{n}^{(k)}(\alpha)=\frac{(-1)^{k} H(\alpha)^{r+k} B_{r}}{(r-1) !(r+k)}
$$


To prove (10), we use (12), (13) and get, for $z \neq \alpha$,

$$
\begin{aligned}
\left|F(z)-g_{n}(z)\right| & \leq\left|u(z)-s_{n, 0}(z)\right|+\sum_{i=1}^{r}\left|B_{i}\right|\left|(z-\alpha)^{-i}-s_{n, i}(z)\right| \\
& \leq\left|\frac{\omega_{n+\mu+\nu}(z)}{\omega_{n+\mu+\nu}(\alpha)}\right|\left[1+\sum_{i=1}^{r}\left|B_{i}\right| \sum_{t=0}^{i-1} \frac{\left|\omega_{n+\mu+\nu}(\alpha) \theta_{n+\mu+\nu}^{(t)}(\alpha)(z-\alpha)^{t-i}\right|}{t}\right] \\
& \leq\left|\frac{\omega_{n+\mu+\nu}(z)}{\omega_{n+\mu+\nu}(\alpha)}\right| L(z) n^{r-1}
\end{aligned}
$$

where $L(z)$ does not depend on $n$.

Note. Define $g_{n, k}(z):=L_{n+\mu+\nu}\left((z-\alpha)^{k-1+\nu} F(z) ; z\right)$ for $k=1,2, \ldots$ Then, by the same reasoning, for $k=1,2, \ldots, r-\nu$,

$$
\lim _{n \rightarrow \infty} n^{-(r-k+1-\nu+j)} g_{n, k}^{(j)}(\alpha)=\frac{(-1)^{j} H(\alpha)^{r-k+1-\nu+j} B_{r}}{(r-k+1-\nu-1) !(r-k+1-\nu+j)} \neq 0 .
$$

If $k>r-\nu$, then $(z-\alpha)^{k-1+\nu} F(z)$ has no pole at $z=\alpha$. If $k=r-\nu+1$, then $(z-\alpha)^{k-1+\nu} F(z)$ is analytic and not zero at $z=\alpha$, thus we have

$$
\begin{gathered}
\lim _{n \rightarrow \infty} n^{-(r-k+1-\nu)} g_{n, k}(\alpha)=\lim _{n \rightarrow \infty} g_{n, k}(\alpha)=B_{r} \neq 0, \\
\lim _{n \rightarrow \infty} n^{-(r-k+1-\nu+j)} g_{n, k}^{(j)}(\alpha)=\lim _{n \rightarrow \infty} n^{-j} g_{n, k}^{(j)}(\alpha)=0, \quad \text { for } j>0 .
\end{gathered}
$$

If $k>r-\nu+1$, then $(z-\alpha)^{k-1+\nu} F(z)$ is analytic and has a zero of order $k-(r-\nu+1)$ at $z=\alpha$. Hence, there exits a $\sigma>\rho$ such that $(z-\alpha)^{k-1+\nu} F(z)$ is analytic in $E_{\sigma}$. By the Hermite formula,

$$
g_{n, k}(z)-(z-\alpha)^{k-1+\nu} F(z)=\frac{1}{2 \pi i} \int_{\Gamma_{\sigma}} \frac{\omega_{n+\mu+\nu}(z)(t-\alpha)^{k-1+\nu} F(t)}{\omega_{n+\mu+\nu}(t)(t-z)} d t, \quad z \in E_{\sigma} .
$$

Thus

$$
\lim _{n \rightarrow \infty} n^{\tau} g_{n, k}^{(j)}(\alpha)=0 \quad \text { for } j=0,1, \ldots, k-(r-\nu+1)-1, \text { and any real } \tau \text {. }
$$

Proof of Theorem 1. By the assumption of Theorem $1, f(z)$ has $\mu$ poles inside $E_{\rho}$ and $m$ different poles $\alpha_{1}, \alpha_{2}, \ldots, \alpha_{m}$ on the $\Gamma_{\rho}$ with multiplicities $r_{1}, r_{2}, \ldots, r_{m}$, respectively, $\left(H\left(\alpha_{i}\right) \neq 0, i=1,2, \ldots, m\right)$. Now, since $\pi(z) f(z)$ is analytic in $E_{\rho}$, write

$$
\begin{gathered}
\pi(z) f(z)=: f_{0}(z)+\sum_{i=1}^{m} \sum_{j=1}^{r_{i}} B_{j, i}\left(z-\alpha_{i}\right)^{-j} \\
\left(z-\alpha_{j}\right)^{k-1+p_{j}} \prod_{i=1, i \neq j}^{m}\left(z-\alpha_{i}\right)^{2 p_{i}} \pi^{2}(z) f(z) \\
=: u_{j}(z)+\sum_{l=1}^{r_{j}-k+1-p_{j}} C_{l, j j}\left(z-\alpha_{j}\right)^{-l}+\sum_{i=1, i \neq j}^{m} \sum_{l=1}^{r_{i}-2 p_{i}} C_{l, j i}\left(z-\alpha_{i}\right)^{-l},
\end{gathered}
$$

$j$ is an integer such that $p_{j}>0$ and $1 \leq j \leq m$, where $f_{0}(z), u_{j}(z)$ are analytic on $E_{\rho}$; if any $r_{j}-k+1-p_{j}, r_{i}-2 p_{i}$ is less than 1 , then the corresponding sum is empty. We define any empty sum to be zero. 
Let $s_{n, l, j}(z):=L_{n+\mu+\nu}\left(\left(z-\alpha_{j}\right)^{-l} ; z\right),(j=1,2, \ldots, m, l>0)$. Then by (11), we get

$$
\begin{aligned}
(-l)(-l-1) & \cdots(-l-q+1)\left(z-\alpha_{j}\right)^{-l-q}-s_{n, l, j}^{(q)}(z)=\sum_{s=0}^{q}\left(\begin{array}{l}
q \\
s
\end{array}\right) \omega_{n+\mu+\nu}^{(q-s)}(z) \\
& \times \sum_{t=0}^{l-1} \theta_{n+\mu+\nu}^{(t)}\left(\alpha_{j}\right)(t-l)(t-l-1) \cdots(t-l-s+1)\left(z-\alpha_{j}\right)^{t-l-s} / t ! .
\end{aligned}
$$

We cannot guarantee the existence of the limit with $M_{1}<\left|\omega_{n+\mu+\nu}\left(\alpha_{i}\right) / \omega_{n+\mu+\nu}\left(\alpha_{j}\right)\right|<$ $M_{2}$ and $\alpha_{i} \neq \alpha_{j}$ when $i \neq j$; however, we have

$$
\limsup _{n \rightarrow \infty} n^{-(l-1+q)}\left|s_{n, l, j}^{(q)}\left(\alpha_{i}\right)\right|=\left|M_{l, q, j i} H^{l-1+q}\left(\alpha_{i}\right)\right|, \quad i \neq j .
$$

For $i=j$, we expand the right side of (12) in a neighborhood of $\alpha_{i}$ and differentiate

$$
\begin{aligned}
s_{n, l, i}^{(q)}(z)=\sum_{s=0}^{q} & \sum_{t=q-s}^{\infty}\left(\begin{array}{l}
q \\
s
\end{array}\right) \\
& \times \frac{\omega_{n+\mu+\nu}^{(s)}(z) \theta_{n+\mu+\nu}^{(t)}\left(\alpha_{i}\right) t(t-1) \cdots(t-q+s+1)\left(z-\alpha_{i}\right)^{t-q+s}}{(t+l) !},
\end{aligned}
$$

thus, we obtain

$$
\begin{aligned}
\lim _{n \rightarrow \infty} n^{-(l+q)} s_{n, l, i}^{(q)}\left(\alpha_{i}\right) & =\lim _{n \rightarrow \infty} n^{-(l+q)} \sum_{s=0}^{q}\left(\begin{array}{l}
q \\
s
\end{array}\right) \frac{\omega_{n+\mu+\nu}^{(q-s)}(z) \theta_{n+\mu+\nu}^{(l+s)}\left(\alpha_{i}\right) s !}{(l+s) !} \\
& =\frac{(-1)^{q} H^{l+q}\left(\alpha_{i}\right)}{(l-1) !(l+q)} .
\end{aligned}
$$

For $j=1,2, \ldots, m$, define

$$
\begin{aligned}
g_{n, k, j}(z):=L_{n+\mu+\nu}\left(\left(z-\alpha_{j}\right)^{k-1+p_{j}} \prod_{i=1, i \neq j}^{m}\left(z-\alpha_{i}\right)^{2 p_{i}} \pi^{2}(z) f(z) ; z\right), & \\
g_{n, p_{1}+1}(z):=L_{n+\mu+\nu}\left(\prod_{i=1}^{m}\left(z-\alpha_{i}\right)^{2 p_{i}} \pi^{2}(z) f(z) ; z\right) . & \text { for } k=1,2, \ldots, p_{j},
\end{aligned}
$$

Then, since $j$ is an integer such that $p_{j}>0$ and $1 \leq j \leq m$,

$$
g_{n, k, j}(z)=s_{n, 0, j}(z)+\sum_{l=1}^{r_{j}-k+1-p_{j}} C_{l, j j} s_{n, l, j}(z)+\sum_{i=1, i \neq j}^{m} \sum_{l=1}^{r_{i}-2 p_{i}} C_{l, j i} s_{n, l, i}(z)
$$

where $s_{n, 0, j}(z):=L_{n+\mu+\nu}\left(u_{j}(z) ; z\right)(j=1,2, \ldots, m)$. Combining this with (18)-(22), if $r_{j}-k+1-p_{j} \geq r_{i}-2 p_{i}, i=1,2, \ldots, m, 1 \leq k \leq p_{j}$, then

$$
\begin{gathered}
\lim _{n \rightarrow \infty} n^{-\left(r_{j}-k-p_{j}+1+s\right)} g_{n, k, j}^{(s)}\left(\alpha_{j}\right)=N_{s, j j}^{\{k\}}<\infty, \\
\limsup _{n \rightarrow \infty} n^{-\max \left\{\left(r_{j}-k-p_{j}+s\right),\left(r_{i}-2 p_{i}+s\right)\right\}}\left|g_{n, k, j}^{(s)}\left(\alpha_{i}\right)\right|=\left|N_{s, j i}^{\{k\}}\right|<\infty, \quad i \neq j .
\end{gathered}
$$

With all these preparations, now we are ready to consider the multipoint Padé approximation of type $(n, \nu)$ for $f(z)$ and $\operatorname{good} \nu, 0 \leq \nu \leq \sum_{i=1}^{m} r_{i}$. We will examine three cases separately. 
Case 1. $0 \leq \nu \leq\left[\left(r_{1}-r_{2}\right) / 2\right]$. All $\nu$ 's are good in this case. Set $p_{1}:=p_{\nu, 1}=\nu$, $p_{j}:=p_{\nu, j}=0, j=2,3, \ldots, m$. Then $r_{1}-2 \nu \geq r_{i}, i=2,3, \ldots, m$, and (23) becomes

$$
\begin{aligned}
\lim _{n \rightarrow \infty} n^{-\left(r_{1}-k-\nu+1+s\right)} g_{n, k, 1}^{(s)}\left(\alpha_{1}\right) & =B_{r_{1}, 1} \frac{(-1)^{r_{1}-k-\nu+1} H^{r_{1}-k-\nu+1+s}\left(\alpha_{1}\right)}{\left(r_{1}-k-\nu+1\right) !\left(r_{1}-k-\nu+1+s\right)} \\
& =: N_{s, 11}^{\{k\}}<\infty .
\end{aligned}
$$

If $\nu=0$, the theorem is true according to Saff (cf. [8]).

We now discuss the case $\nu \geq 1$. Suppose $\mu=0$. Let

$q_{n}(z)=\sum_{k=1}^{\nu} a_{k}^{(n)}\left(z-\alpha_{1}\right)^{k-1}+\left(z-\alpha_{1}\right)^{\nu} \quad$ and $\quad h_{n}(z)=\sum_{k=1}^{\nu} a_{k}^{(n)} g_{n, k, 1}(z)+g_{n, \nu+1}(z)$;

then $h_{n}(z)=: L_{n+\nu}\left(q_{n}(z)\left(z-\alpha_{1}\right)^{\nu} f(z) ; z\right)$. We shall choose the proper coefficients $a_{k}^{(n)}, 1 \leq k \leq \nu$, so that the polynomial $\left(z-\alpha_{1}\right)^{\nu}$ is a factor of $h_{n}(z)$. For this purpose, we need to find a non-trivial solution $\left(a_{1}^{(n)}, a_{2}^{(n)}, \ldots, a_{\nu}^{(n)}\right)^{T}$ for the linear system

$$
\sum_{k=1}^{\nu} a_{k}^{(n)} g_{n, k, 1}^{(j)}\left(\alpha_{1}\right)=-g_{n, \nu+1}^{(j)}\left(\alpha_{1}\right), \quad j=0,1, \ldots, \nu-1 .
$$

To save space, we denote $M_{t \times s}=\left(m_{j, k}\right)_{j=1,2, \ldots, t ; k=1,2, \ldots, s}$ for a matrix with $t$ rows and $s$ columns. Let

$$
\begin{gathered}
A_{n, \nu \times \nu}=\left(g_{n, k, 1}^{(j-1)}\left(\alpha_{1}\right)\right)_{j=1,2, \ldots, \nu ; k=1,2, \ldots, \nu}, \\
A_{n, \nu \times \nu}^{\star}:=n^{-\nu\left(r_{1}-\nu\right)} A_{n, \nu \times \nu} \\
=\left(n^{-\left(r_{1}-\nu+j-1-k+1\right)} g_{n, k, 1}^{(j-1)}\left(\alpha_{1}\right)\right)_{j=1,2, \ldots, \nu ; k=1,2, \ldots, \nu} \\
Y_{n}:=\left(g_{n, \nu+1,1}^{(j-1)}\left(\alpha_{1}\right)\right)_{j=1,2, \ldots, \nu}, \\
Y_{n}^{\star}:=\left(n^{-\left(r_{1}-2 \nu+j-1\right)} g_{n, \nu+1,1}^{(j-1)}\left(\alpha_{1}\right)\right)_{j=1,2, \ldots, \nu} .
\end{gathered}
$$

Noticing that $r_{1}-2 \nu \geq r_{2}>0$ and $r_{1}-\nu>\nu$ by (25), we obtain

$$
\begin{aligned}
\lim _{n \rightarrow \infty} n^{-\nu\left(r_{1}-\nu\right)} \operatorname{det}\left(A_{n, \nu \times \nu}\right) & =\lim _{n \rightarrow \infty} \operatorname{det}\left(A_{n, \nu \times \nu}^{\star}\right)=d_{\nu} D_{\nu} \neq 0, \\
\lim _{n \rightarrow \infty} Y_{n}^{\star} & =\left(N_{j-1,11}^{\{\nu+1\}}\right)_{j=1,2, \ldots, \nu}
\end{aligned}
$$

where the specific form of the constant $d_{\nu}$ and the non-zero determinant $D_{\nu}$ are given in [5]. Thus, for $n$ large enough, $\operatorname{det}\left(A_{n, \nu \times \nu}\right) \neq 0$. Hence, the linear system (26) has the unique solution for sufficiently large $n$. Furthermore, $X_{n}^{\star}:=\left(n^{\nu} a_{1}^{(n)}, n^{\nu-1} a_{2}^{(n)}, \ldots\right.$, $\left.n^{1} a_{\nu}^{(n)}\right)^{T}$ satisfying $A_{n, \nu \times \nu}^{\star} X_{n}^{\star}=Y_{n}^{\star}$, and it has a finite limit

$$
n^{\nu-k+1} a_{k}^{(n)} \rightarrow \frac{d_{\nu}^{\{k\}} D_{\nu}^{\{k\}} H\left(\alpha_{1}\right)^{k-\nu-1}}{d_{\nu} D_{\nu}}<\infty, \quad \text { for } k=1,2, \ldots, \nu,
$$

where $d_{\nu}^{\{k\}}$ and $D_{\nu}^{\{k\}}$ are constants depending only on $k$ and $\nu$. From this, we see that for each compact set $Q$ of the plane

$$
\left\|q_{n}(z)-\left(z-\alpha_{1}\right)^{\nu}\right\|_{Q} \leq C / n
$$

Now set $R_{n, \nu}(z):=h_{n}(z) /\left\{q_{n}(z)\left(z-\alpha_{1}\right)^{\nu}\right\}$. Then, by our choice of the coefficients $a_{k}^{(n)}$ 's, we know that $R_{n, \nu}(z)$ is a rational function of type $(n, \nu)$, and $q_{n}(z)$ is not zero 
inside $E$ for $n$ large enough from (29). Hence, $R_{n, \nu}(z)$ must interpolate $f(z)$ on the set $\Lambda(n+\nu)$. Moreover, from Lemma 1

$$
\begin{aligned}
\mid f(z) & -\frac{h_{n}(z)}{q_{n}(z)\left(z-\alpha_{1}\right)^{\nu}} \mid \\
& \leq \frac{\sum_{k=1}^{\nu}\left|a_{k}^{(n)}\right|\left|\left(z-\alpha_{1}\right)^{k-1+\nu} f(z)-g_{n, k}(z)\right|+\left|\left(z-\alpha_{1}\right)^{2 \nu} f(z)-g_{n, \nu+1}(z)\right|}{\left|q_{n}(z)\left(z-\alpha_{1}\right)^{\nu}\right|} \\
& \leq M n^{r-2 \nu-1} \sigma^{n} / \rho^{n} \quad \text { for } z \text { on } E_{\sigma} \subset E_{\rho} .
\end{aligned}
$$

We have proved the results for $\mu=0$.

Next, suppose $\mu>0$ and $\gamma_{1}, \gamma_{2}, \ldots, \gamma_{\mu}$ are poles of $f(z)$ inside $E_{\rho}$ with $\left|\gamma_{1}\right| \leq\left|\gamma_{2}\right| \leq$ $\cdots \leq\left|\gamma_{\mu}\right|$. Define $\pi_{j}(z):=\prod_{t=1}^{j}\left(z-\gamma_{t}\right), j=1,2, \ldots, \mu$, and $\pi_{0}(z):=1$. It is obvious that $\pi_{\mu}(z) \equiv \pi(z)$. Define

$$
w_{n, s}(z):=L_{n+\mu+\nu}\left(\pi_{s-1}(z) \pi(z)\left(z-\alpha_{1}\right)^{2 \nu} f(z) ; z\right) \quad \text { for } s=1,2, \ldots, \mu .
$$

Let

$$
q_{n}(z)=\sum_{s=1}^{\mu} b_{s}^{(n)} \pi_{s-1}(z)\left(z-\alpha_{1}\right)^{\nu}+\sum_{k=1}^{\nu} a_{k}^{(n)}\left(z-\alpha_{1}\right)^{k-1} \pi(z)+\left(z-\alpha_{1}\right)^{\nu} \pi(z) .
$$

Then

$$
\begin{aligned}
h_{n}(z) & =\sum_{s=1}^{\mu} b_{s}^{(n)} w_{n, s}(z)+\sum_{k=1}^{\nu} a_{k}^{(n)} g_{n, k, 1}(z)+g_{n, \nu+1}(z) \\
& =: L_{n+\mu+\nu}\left(q_{n}(z) \pi(z)\left(z-\alpha_{1}\right)^{\nu} f(z) ; z\right) .
\end{aligned}
$$

Now we need $h_{n}(z)=\pi(z)\left(z-\alpha_{1}\right)^{\nu} d_{n}(z)$ where $d_{n}(z)$ is a polynomial of degree $\leq n$. Thus, we get

$$
\left(\begin{array}{ll}
C_{n, \mu \times \mu} & U_{n, \mu \times \nu} \\
V_{n, \nu \times \mu} & A_{n, \nu \times \nu}
\end{array}\right) X_{n}=Y_{n}
$$

where the entries are as follows. Suppose we have $\iota$ different $\gamma$ 's, the multiplicities are $i_{1}, i_{2}, \ldots, i_{\iota}$, respectively $\left(i_{1}+i_{2}+\cdots+i_{\iota}=\mu\right)$. Let $y_{1}=-g_{n, \nu+1}\left(\gamma_{1}\right), \ldots, y_{i_{1}}=$ $-g_{n, \nu+1}^{\left(i_{1}-1\right)}\left(\gamma_{1}\right), \ldots, y_{i_{1}+i_{2}+\cdots+i_{\iota-1}+1}=-g_{n, \nu+1}\left(\gamma_{\mu}\right), \ldots, y_{i_{1}+i_{2}+\cdots+i_{\iota}}=-g_{n, \nu+1}^{\left(i_{\iota}-1\right)}\left(\gamma_{\mu}\right)$; $y_{\mu+k}=-g_{n, \nu+1}^{(k-1)}\left(\alpha_{1}\right)$ for $k=1,2, \ldots, \nu$;

$$
\begin{gathered}
X_{n}=\left(b_{1}^{(n)}, b_{2}^{(n)}, \ldots, b_{\mu}^{(n)}, a_{1}^{(n)}, a_{2}^{(n)}, \ldots, a_{\nu}^{(n)}\right)^{\mathrm{T}} ; \\
Y_{n}:=\left(y_{1}, y_{2}, \ldots, y_{\mu}, y_{\mu+1}, \ldots, y_{\mu+\nu}\right)^{\mathrm{T}} ; \\
C_{n, \mu \times \mu}:=\left(\begin{array}{cccc}
w_{n, 1}\left(\gamma_{1}\right) & w_{n, 2}\left(\gamma_{1}\right) & \ldots & w_{n, \mu}\left(\gamma_{1}\right) \\
\vdots & \vdots & & \vdots \\
w_{n, 1}^{\left(i_{1}-1\right)}\left(\gamma_{1}\right) & w_{n, 2}^{\left(i_{1}-1\right)}\left(\gamma_{1}\right) & \cdots & w_{n, \mu}^{\left(i_{1}-1\right)}\left(\gamma_{1}\right) \\
\vdots & \vdots & & \vdots \\
w_{n, 1}\left(\gamma_{\mu}\right) & w_{n, 2}\left(\gamma_{\mu}\right) & \ldots & w_{n, \mu}\left(\gamma_{\mu}\right) \\
\vdots & \vdots & & \vdots \\
w_{n, 1}^{\left(i_{\iota}-1\right)}\left(\gamma_{\mu}\right) & w_{n, 2}^{\left(i_{\iota}-1\right)}\left(\gamma_{\mu}\right) & \cdots & w_{n, \mu}^{\left(i_{\iota}-1\right)}\left(\gamma_{\mu}\right)
\end{array}\right)
\end{gathered}
$$




$$
U_{n, \mu \times \nu}:=\left(\begin{array}{cccc}
g_{n, 1}\left(\gamma_{1}\right) & g_{n, 2}\left(\gamma_{1}\right) & \cdots & g_{n, \nu}\left(\gamma_{1}\right) \\
\vdots & \vdots & & \vdots \\
g_{n, 1}^{\left(i_{1}-1\right)}\left(\gamma_{1}\right) & g_{n, 2}^{\left(i_{1}-1\right)}\left(\gamma_{1}\right) & \cdots & g_{n, \nu}^{\left(i_{1}-1\right)}\left(\gamma_{1}\right) \\
\vdots & \vdots & & \vdots \\
g_{n, 1}\left(\gamma_{\mu}\right) & g_{n, 2}\left(\gamma_{\mu}\right) & \cdots & g_{n, \nu}\left(\gamma_{\mu}\right) \\
\vdots & \vdots & & \vdots \\
g_{n, 1}^{\left(i_{\iota}-1\right)}\left(\gamma_{\mu}\right) & g_{n, 2}^{\left(i_{\iota}-1\right)}\left(\gamma_{\mu}\right) & \cdots & g_{n, \nu}^{\left(i_{\iota}-1\right)}\left(\gamma_{\mu}\right)
\end{array}\right)
$$

and $A_{n, \nu \times \nu}$ is defined as in (27). It is obvious that $C_{n, \mu \times \mu}$ tend to a lower triangular matrix with non-zero diagonal elements as $n$ tends to infinity, $\operatorname{det}\left(C_{n, \mu \times \mu}\right) \neq 0$ for $n$ sufficiently large.

If we let $X_{n}^{\star}=\left(n^{\tau} b_{1}^{(n)}, n^{\tau} b_{2}^{(n)}, \ldots, n^{\tau} b_{\mu}^{(n)}, n^{\nu} a_{1}^{(n)}, n^{\nu-1} a_{2}^{(n)}, \ldots, n a_{\nu}^{(n)}\right)^{\mathrm{T}}$ for some positive constant $\tau$, it satisfies

$$
\left(\begin{array}{cc}
C_{n, \mu \times \mu} & U_{n, \mu \times \nu}^{\star} \\
V_{n, \nu \times \mu}^{\star} & A_{n, \nu \times \nu}^{\star}
\end{array}\right) X_{n}^{\star}=Y_{n}^{\star}
$$

where $U_{n, \mu \times \nu}^{\star}$ is obtained by multiplying the $k$ th column of $U_{n, \mu \times \nu}$ with $n^{(\tau-\nu-1+k)}$; $V_{n, \nu \times \mu}^{\star}$ is obtained by multiplying the $k$ th row of $V_{n, \nu \times \mu}$ with $n^{-\left(r_{1}-2 \nu+\tau+k-1\right)} ; A_{n, \nu \times \nu}^{\star}$ is defined as before; and

$$
Y_{n}^{\star}=\left(n^{\tau} y_{1}, n^{\tau} y_{2}, \ldots, n^{\tau} y_{\mu}, n^{-\left(r_{1}-2 \nu\right)} y_{\mu+1}, \ldots, n^{-\left(r_{1}-\nu-1\right)} y_{\mu+\nu}\right)^{\mathrm{T}} .
$$

Because all $g_{n, k, 1}(z)$ 's are analytic and tend to zero at $\gamma_{t}$ 's, by (20), we see that $U_{n, \mu \times \nu}^{\star}$ tends to a zero matrix as $n \rightarrow \infty$ for $\nu$ and any real $\tau$. Furthermore, by the same reasoning as the proof of (17), we know that

$$
\lim _{n \rightarrow \infty} n^{-\left(r_{1}-2 \nu+j\right)} \omega_{n, s}^{(j)}\left(\alpha_{1}\right)=\frac{(-1)^{j} H\left(\alpha_{1}\right)^{r_{1}-2 \nu+j} B_{r_{1}} \pi_{s-1}\left(\alpha_{1}\right) \pi\left(\alpha_{1}\right)}{\left(r_{1}-2 \nu-1\right) !\left(r_{1}-2 \nu+j\right)} \text { for } 2 \nu<r_{1} .
$$

Thus $\lim _{n \rightarrow \infty} V_{n, \nu \times \mu}^{\star}=V_{\nu \times \mu}$, which is a finite matrix for $\tau=0$ and a zero matrix for $\tau>0$ and

$$
\lim _{n \rightarrow \infty} Y_{n}^{\star}=\left(0,0, \ldots, 0, N_{0,11}^{\{\nu+1\}}, N_{1,11}^{\{\nu+1\}}, \ldots, N_{\nu-1,11}^{\{\nu+1\}}\right)^{\mathrm{T}} .
$$

By the previous proof for $\mu=0$, we know that for $n$ large enough, $\operatorname{det}\left(A_{n, \nu \times \nu}^{\star}\right) \neq 0$, hence the linear system (30) has a unique solution and $\lim _{n \rightarrow \infty} X_{n}^{\star}$ exists and is bounded. That means $h_{n}(z) /\left\{\pi(z)\left(z-\alpha_{1}\right)^{\nu} q_{n}(z)\right\}$ is a rational function of type $(n, \mu+\nu)$ which interpolates $f(z)$ on the set $\Lambda(n+\mu+\nu)$ and (4) is true. Furthermore, we have the estimate

$$
\begin{aligned}
f(z)-\frac{h_{n}(z)}{q_{n}(z) \pi(z)(z-} & \left.\alpha_{1}\right)^{\nu} \\
& =\frac{\omega_{n+\mu+\nu}(z)}{q_{n}(z) \pi(z)\left(z-\alpha_{1}\right)^{\nu}} \\
& \times \frac{H\left(\alpha_{1}\right)^{r_{1}-2 \nu-1} n^{r_{1}-2 \nu-1} B_{r_{1}}}{\omega_{n+\mu+\nu}\left(\alpha_{1}\right) D_{\nu}\left(r_{1}-2 \nu-1\right) !}\left\{C_{\nu}+o(1)+O\left(n^{-\tau}\right)\right\}
\end{aligned}
$$

with a non-zero determinant $C_{\nu}$. Hence, (3) holds for both $\mu=0$ and $\mu>0$.

Case 2. $v_{l-1} \leq \nu<v_{l}$ when $2 \leq l \leq m-1$ or $v_{m-1} \leq \nu \leq v_{m}$ when $l=m$. If $\nu$ is good, set $p_{i}=\bar{p}_{\nu, i}, i=1,2, \ldots, m$; then by Definitions 1 and 2 , we get (cf. [6] for the 
proof)

$$
r_{j}-2 p_{j}+1>r_{i}-2 p_{i}-1 \quad \text { for } 1 \leq j \leq l \text { and } i=1,2, \ldots, m .
$$

Thus (17)-(20) become

$$
\lim _{n \rightarrow \infty} n^{-\left(r_{j}-k-p_{j}+1+s\right)} g_{n, k, j}^{(s)}\left(\alpha_{j}\right)=N_{s, j j}^{\{k\}}, \quad k=1,2, \ldots, p_{j}, j=1, \ldots, l,
$$

where, for $j=1, \ldots, l$,

$$
N_{s, j j}^{\{k\}}=\left\{\begin{array}{lc}
\frac{(-1)^{s} H\left(\alpha_{j}\right)^{r_{j}-k+1-p_{j}+s} B_{r_{j}, j}}{\left(r_{j}-k+1-p_{j}-1\right) !\left(r_{j}-k+1-p_{j}+s\right)}, & \text { if } k \leq r_{j}-p_{j}, \\
B_{r_{j}, j}, & s=0,1, \ldots, p_{j} ; \\
0, & \text { if } k=r_{j}-p_{j}+1, \\
& s=0 ; \\
0, & \text { if } k=r_{j}-p_{j}+1, \\
& s=1, \ldots, p_{j} ; \\
& \text { if } k>r_{j}-p_{j}+1, \\
& s=0,1, \ldots, p_{j} .
\end{array}\right.
$$

Notice that $p_{i}=0$ for $l+1 \leq i \leq m$; let

$$
w_{n, s}(z):=L_{n+\mu+\nu}\left(\prod_{i=1}^{m}\left(z-\alpha_{i}\right)^{2 p_{i}} \pi_{s-1}(z) \pi(z) f(z) ; z\right), \quad s=1,2, \ldots, \mu,
$$

and construct

$$
\begin{aligned}
q_{n}(z)= & \sum_{j=1}^{l} \sum_{k=1}^{p_{j}} a_{k, j}^{(n)}\left(z-\alpha_{j}\right)^{k-1} \prod_{i=1, i \neq j}^{l}\left(z-\alpha_{i}\right)^{p_{i}} \pi(z) \\
& +\sum_{s=1}^{\mu} b_{s}^{(n)} \pi_{s-1}(z) \prod_{i=1}^{l}\left(z-\alpha_{i}\right)^{p_{i}}+\pi(z) \prod_{i=1}^{l}\left(z-\alpha_{i}\right)^{p_{i}}, \\
h_{n}(z)= & \sum_{j=1}^{l} \sum_{k=1}^{p_{j}} a_{k, j}^{(n)} g_{n, k, j}(z)+\sum_{s=1}^{\mu} b_{s}^{(n)} w_{n, s}(z)+g_{n, p_{1}+1}(z) .
\end{aligned}
$$

We want $h_{n}(z)=\prod_{i=1}^{l}\left(z-\alpha_{i}\right)^{p_{i}} \pi(z) d_{n}(z)$ where $d_{n}(z)$ is a polynomial of degree $\leq n$, so we have

$$
\tilde{M}_{n, l+1 \times l+1} X_{n}=Y_{n}
$$

where $\tilde{M}_{n, l+1 \times l+1}$ is a matrix of matrices with entries

$$
\begin{aligned}
& M_{n, j, i}:=\left(g_{n, k, i}^{(s)}\left(\alpha_{j}\right)\right)_{s=0,1, \ldots, p_{j}-1 ; k=1,2, \ldots, p_{i}}, \quad i, j=1, \ldots, l ; \\
& M_{n, j, l+1}:=\left(w_{n, k}^{(s)}\left(\alpha_{j}\right)\right)_{s=0,1, \ldots, p_{j}-1 ; k=1,2, \ldots, \mu}, \quad j=1, \ldots, l \text {; } \\
& M_{n, l+1, i}:=\left(\begin{array}{cccc}
g_{n, 1, i}\left(\gamma_{1}\right) & g_{n, 2, i}\left(\gamma_{1}\right) & \cdots & g_{n, p_{i}, i}\left(\gamma_{1}\right) \\
\vdots & \vdots & & \vdots \\
g_{n, 1, i}^{\left(i_{1}-1\right)}\left(\gamma_{1}\right) & g_{n, 2, i}^{\left(i_{1}-1\right)}\left(\gamma_{1}\right) & \cdots & g_{n, p_{i}, i}^{\left(i_{1}-1\right)}\left(\gamma_{1}\right) \\
\vdots & \vdots & & \vdots \\
g_{n, 1, i}\left(\gamma_{\mu}\right) & g_{n, 2, i}\left(\gamma_{\mu}\right) & \cdots & g_{n, p_{i}, i}\left(\gamma_{\mu}\right) \\
\vdots & \vdots & & \vdots \\
g_{n, 1, i}^{\left(i_{2}-1\right)}\left(\gamma_{\mu}\right) & g_{n, 2, i}^{\left(i_{2}-1\right)}\left(\gamma_{\mu}\right) & \cdots & g_{n, p_{i}, i}^{\left(i_{2}-1\right)}\left(\gamma_{\mu}\right)
\end{array}\right)
\end{aligned}
$$




$$
\begin{gathered}
M_{n, l+1, l+1}:=C_{n, \mu \times \mu} ; \\
X_{n}=\left(a_{1,1}^{(n)}, a_{2,1}^{(n)}, \ldots, a_{p_{1}, 1}^{(n)}, \ldots, a_{1, l}^{(n)}, a_{2, l}^{(n)}, \ldots, a_{p_{l}, l}^{(n)}, b_{1}^{(n)}, b_{2}^{(n)}, \ldots, b_{\mu}^{(n)}\right)^{\mathrm{T}}, \\
Y_{n}=\left(-g_{n, p_{1}+1}\left(\alpha_{1}\right),-g_{n, p_{1}+1}^{\prime}\left(\alpha_{1}\right), \ldots,-g_{n, p_{1}+1}^{\left(p_{1}-1\right)}\left(\alpha_{1}\right),\right. \\
\quad \ldots,-g_{n, p_{1}+1}\left(\alpha_{l}\right),-g_{n, p_{1}+1}^{\prime}\left(\alpha_{l}\right), \ldots,-g_{n, p_{1}+1}^{\left(p_{l}-1\right)}\left(\alpha_{l}\right), \\
\left.\quad-g_{n, p_{1}+1}\left(\gamma_{1}\right), \ldots,-g_{n, p_{1}+1}^{\left(i_{1}-1\right)}\left(\gamma_{1}\right), \ldots,-g_{n, p_{1}+1}\left(\gamma_{\mu}\right), \ldots,-g_{n, p_{1}+1}^{\left(i_{1}-1\right)}\left(\gamma_{\mu}\right)\right)^{\mathrm{T}} .
\end{gathered}
$$

Let $\tilde{N}_{l+1 \times l+1}$ be a diagonal matrix of matrices with diagonal entries $N_{i, i}$, let $\tilde{W}_{l+1 \times l+1}$ be another diagonal matrix of matrices with its diagonal elements $W_{i, i}$ where, for $i=1,2, \ldots, l$,

$$
\begin{gathered}
N_{i, i}=\left(\begin{array}{cccc}
n^{-\left(r_{i}-2 p_{i}\right)} & & & \\
& n^{-\left(r_{i}-2 p_{i}+1\right)} & & \\
& & \ddots & \\
& & & n^{-\left(r_{i}-p_{i}-1\right)}
\end{array}\right), \\
W_{i, i}=\left(\begin{array}{cccc}
n^{-p_{i}} & & & \\
& n^{-p_{i}+1} & & \\
& & \ddots & \\
& & & n^{-1}
\end{array}\right),
\end{gathered}
$$

$N_{l+1, l+1}:=n^{\tau} I_{\mu},\left(I_{\mu}\right.$ is the identity matrix of order $\left.\mu\right)$, and $W_{l+1, l+1}:=N_{l+1, l+1}^{-1}$. Then

$$
\begin{aligned}
& \tilde{M}_{n, l+1 \times l+1}^{\star}:=\tilde{N}_{l+1 \times l+1} \cdot \tilde{M}_{n, l+1 \times l+1} \cdot \tilde{W}_{l+1 \times l+1}, \\
& X_{n}^{\star}=\tilde{W}_{l+1 \times l+1}^{-1} \cdot X_{n 2} \text { and } Y_{n}^{\star}=\tilde{N}_{l+1 \times l+1} \cdot Y_{n}
\end{aligned}
$$

satisfy

$$
\tilde{M}_{n, l+1 \times l+1}^{\star} X_{n}^{\star}=Y_{n}^{\star} .
$$

Let $M_{i, i}^{\star}=\lim _{n \rightarrow \infty} M_{n, i, i}^{\star}$. By (33), we obtain, for $i=1, \ldots, l$,

$$
\operatorname{det}\left(M_{i, i}^{\star}\right):=\lim _{n \rightarrow \infty} \operatorname{det}\left(M_{n, i, i}^{\star}\right)=\lim _{n \rightarrow \infty} n^{-p_{i}\left(r_{i}-p_{i}\right)} \operatorname{det}\left(M_{n, i, i}\right)=d_{p_{i}, i} D_{p_{i}, i} \neq 0 .
$$

Similar to the reasoning in the proof of Case 1, we obtain for any $\tau>0$,

$$
\begin{aligned}
M_{j, l+1}^{\star}:=\lim _{n \rightarrow \infty} M_{n, j, l+1}^{\star}=V_{p_{j} \times \mu}, & j=1, \ldots, l ; \\
M_{l+1, i}^{\star}:=\lim _{n \rightarrow \infty} M_{n, l+1, i}^{\star}=O_{\mu \times p_{i}}, & i=1, \ldots, l .
\end{aligned}
$$

As for the limits of $M_{n, i, j}$ where $i \neq j$, we need further discussions. If $r_{i}-2 p_{i} \leq 0$ for $1 \leq i \leq m$ and $r_{j}-p_{j}-k+1 \leq 0$, then $g_{n, k, j}(z)$ interpolates an analytic function. Furthermore, the interpolated function $\left(z-\alpha_{j}\right)^{k-1+p_{j}} \prod_{t=1, t \neq j}^{m}\left(z-\alpha_{t}\right)^{2 p_{t}} \pi(z) f(z)$ has a zero of order $k-1-r_{j}+p_{j}$ at $z=\alpha_{j}$ and a zero of order $2 p_{i}-r_{i}$ at $z=\alpha_{i}, i \neq j$, $1 \leq i \leq m$. Hence, we have

$$
\lim _{n \rightarrow \infty} n^{-\left(r_{i}-2 p_{i}+p_{j}-k+1+s\right)} g_{n, k, j}^{(s)}\left(\alpha_{i}\right)=0,
$$

by (20) for $s=0,1, \ldots, 2 p_{i}-r_{i}-1$ and by $(18),(19)$ for $s \geq 2 p_{i}-r_{i}$. On the other hand, if $r_{i}-2 p_{i} \leq 0$ for $1 \leq i \leq m$ and $r_{j}-p_{j}-k+1>0$, then the interpolated function has a pole of order $k-1-r_{j}-p_{j}$ at $z=\alpha_{j}$. If one of $r_{i}-2 p_{i}>0$, then all $r_{j}-p_{j}-k+1>0$ for $1 \leq k \leq p_{j}$ according to (32). The interpolated function has at 
least one pole. Hence, the elements of $M_{n, j, i}^{\star}$ may not tend to zero; however, they are bounded. By (32), we get

$$
r_{i}-2 p_{i}+1 \geq r_{j}-2 p_{j} \geq r_{i}-2 p_{i}-1 \text { for } 1 \leq i, j \leq l,
$$

so we have three possibilities: $r_{i}-2 p_{i}+1=r_{j}-2 p_{j} ; r_{i}-2 p_{i}=r_{j}-2 p_{j} ; r_{i}-2 p_{i}-1=$ $r_{j}-2 p_{j}$. Let us study them one by one.

(a) $r_{i}-2 p_{i}+1=r_{j}-2 p_{j}$. In this case

$$
\begin{aligned}
& \max \left\{\left(r_{i}-2 p_{i}+s\right),\left(r_{j}-p_{j}-k+s\right)\right\}=r_{j}-p_{j}-k+s, \\
& \max \left\{\left(r_{i}-p_{i}-k+s\right),\left(r_{j}-2 p_{j}+s\right)\right\} \\
& \quad= \begin{cases}r_{i}-p_{i}-k+s & \text { for } 1 \leq k \leq p_{i}-1, \\
r_{j}-2 p_{j}+s=r_{i}-2 p_{i}+1+s & \text { for } k=p_{i} .\end{cases}
\end{aligned}
$$

Thus by (24), the elements of $M_{n, j, i}^{\star}$ tend to a limit:

$$
\begin{gathered}
\lim _{n \rightarrow \infty} n^{-\left(r_{j}-2 p_{j}+p_{i}-k+1+s\right)} g_{n, k, i}^{(s)}\left(\alpha_{j}\right)=\lim _{n \rightarrow \infty} n^{-\left(r_{i}-2 p_{i}+1+p_{i}-k+1+s\right)} g_{n, k, j}^{(s)}\left(\alpha_{j}\right) \\
=\lim _{n \rightarrow \infty} n^{-2} n^{-\left(r_{i}-p_{i}-k+s\right)} g_{n, k, i}^{(s)}\left(\alpha_{j}\right)=0, \quad 1 \leq k \leq p_{i}, \quad i \neq j ;
\end{gathered}
$$

on the other hand, the elements of $M_{n, i, j}^{\star}$ are bounded:

$$
\begin{gathered}
\limsup _{n \rightarrow \infty} n^{-\left(r_{i}-2 p_{i}+p_{j}-k+1+s\right)}\left|g_{n, k, j}^{(s)}\left(\alpha_{i}\right)\right|=\limsup _{n \rightarrow \infty} n^{-\left(r_{j}-2 p_{j}-1+p_{j}-k+1+s\right)}\left|g_{n, k, j}^{(j)}\left(\alpha_{i}\right)\right| \\
=\limsup _{n \rightarrow \infty} n^{-\left(r_{j}-p_{j}-k+s\right)}\left|g_{n, k, j}^{(s)}\left(\alpha_{i}\right)\right|=\left|N_{s, j i}^{\{k\}}\right|, \quad 1 \leq k \leq p_{j}, \quad i \neq j .
\end{gathered}
$$

This means that

$$
\lim _{n \rightarrow \infty} M_{n, j, i}^{\star}=O_{p_{j} \times p_{i}}, \quad \limsup _{n \rightarrow \infty} M_{n, i, j}^{\star}=U_{p_{i} \times p_{j}}^{\star}, \quad \liminf _{n \rightarrow \infty} M_{n, i, j}^{\star}=V_{p_{i} \times p_{j}}^{\star},
$$

where $U_{p_{i} \times p_{j}}^{\star}$ and $V_{p_{i} \times p_{j}}^{\star}$ are finite matrices and $O_{p \times q}$ is a zero matrix.

(b) For the case $r_{i}-2 p_{i}-1=r_{j}-2 p_{j}$, we only need to exchange the positions of $i$ and $j$.

(c) $r_{i}-2 p_{i}=r_{j}-2 p_{j}$. In this case

$$
\begin{aligned}
& \max \left\{\left(r_{i}-p_{i}-k+s\right),\left(r_{j}-2 p_{j}+s\right)\right\}=r_{i}-p_{i}-k+s \quad \text { for } 1 \leq k \leq p_{i}, \\
& \max \left\{\left(r_{i}-2 p_{i}+s\right),\left(r_{j}-p_{j}-k+s\right)\right\}=r_{j}-p_{j}-k+s \quad \text { for } 1 \leq k \leq p_{j} .
\end{aligned}
$$

Thus,

$$
\begin{aligned}
& \lim _{n \rightarrow \infty} n^{-\left(r_{j}-2 p_{j}+p_{i}-k+1+s\right)} g_{n, k, i}^{(s)}\left(\alpha_{j}\right)=\lim _{n \rightarrow \infty} n^{-\left(r_{i}-2 p_{i}+p_{i}-k+1+s\right)} g_{n, k, i}^{(s)}\left(\alpha_{j}\right)=0, i \neq j, \\
& \lim _{n \rightarrow \infty} n^{-\left(r_{i}-2 p_{i}+p_{j}-k+1+s\right)} g_{n, k, j}^{(s)}\left(\alpha_{i}\right)=\lim _{n \rightarrow \infty} n^{-\left(r_{j}-2 p_{j}+p_{j}-k+1+s\right)} g_{n, k, j}^{(s)}\left(\alpha_{i}\right)=0, i \neq j .
\end{aligned}
$$

Hence, if $r_{i}-2 p_{i}=r_{j}-2 p_{j}$ and $i \neq j$, then

$$
\lim _{n \rightarrow \infty} M_{n, j, i}^{\star}=O_{p_{j} \times p_{i}}, \quad \quad \lim _{n \rightarrow \infty} M_{n, i, j}^{\star}=O_{p_{i} \times p_{j}} .
$$

Generally speaking, we are not sure of the existence of $\lim _{n \rightarrow \infty} M_{n, i, j}^{\star}$ for $i \neq j$. However, for every subsequence there exists a convergent sub-subsequence. Let $M_{i, j}^{\star}$ be one limit of $\left\{M_{n, i, j}^{\star}\right\}$. Furthermore, let $\tilde{M}_{l+1 \times l+1}^{\star}$ be one limit of $\left\{\tilde{M}_{n, l+1 \times l+1}^{\star}\right\}$. Then all entries of $\tilde{M}_{l+1 \times l+1}^{\star}$ are bounded; some are zero matrices. We state that

$$
\operatorname{det}\left(\tilde{M}_{l+1 \times l+1}^{\star}\right)=\prod_{i=1}^{l} \operatorname{det}\left(M_{i, i}^{\star}\right) \cdot \operatorname{det} C_{\mu \times \mu} .
$$


To verify this statement, we let $c_{i}=r_{i}-2 p_{i}$ for $1 \leq i \leq l$, and by (32), they satisfy

$$
-1 \leq c_{i}-c_{j} \leq 1 \quad \text { for } 1 \leq i, j \leq l .
$$

Without loss of generality, we assume that (we can always rearrange $c_{1}, c_{2}, \ldots, c_{l}$ in such an order that)

$$
c_{2}-c_{1}=1, \ldots, c_{t}-c_{1}=1, c_{t+1}-c_{1}=0, \ldots, c_{l}-c_{1}=0,
$$

where $t$ is a number between 1 and $l$ and $t=1$ means all $c_{i}^{\prime}$ s are the same. In fact, if $c_{2}-c_{1}=1$, then $c_{i}-c_{1} \neq-1$ because $c_{2}-c_{i} \neq 2$. Thus, the above group of relations is valid. If $t=1$, then all $M_{i, j}^{\star}=O_{p_{i} \times p_{j}}$ for $i \neq j$; we are done with our verification. If $t>1$, for this arrangement, $M_{j, 1}^{\star}=O_{p_{j} \times p_{1}}$ for $j=2, \ldots, l$ by (40) and (41). Thus

$$
\operatorname{det}\left(\tilde{M}_{l+1 \times l+1}^{\star}\right)=\operatorname{det} M_{1,1}^{\star} \operatorname{det}\left(\begin{array}{ccc}
M_{2,2}^{\star} & \cdots & M_{2, l}^{\star} \\
\vdots & & \vdots \\
M_{l, 2}^{\star} & \cdots & M_{l, l}^{\star}
\end{array}\right) \operatorname{det} C_{\mu \times \mu} .
$$

Since

$$
c_{3}-c_{2}=0, \ldots, c_{t}-c_{2}=0, c_{t+1}-c_{2}=-1, \ldots, c_{l}-c_{2}=-1,
$$

$M_{2, j}^{\star}=O_{p_{2} \times p_{j}}$ for $j=3, \ldots, l$ by $(40)$ and (41). Thus

$$
\operatorname{det}\left(\tilde{M}_{l+1 \times l+1}^{\star}\right)=\operatorname{det} M_{1,1}^{\star} \operatorname{det} M_{2,2}^{\star} \operatorname{det}\left(\begin{array}{ccc}
M_{3,3}^{\star} & \cdots & M_{3, l}^{\star} \\
\vdots & & \vdots \\
M_{l, 3}^{\star} & \cdots & M_{l, l}^{\star}
\end{array}\right) \operatorname{det} C_{\mu \times \mu} .
$$

By repeating these steps, we get (42). Hence,

$$
\lim _{n \rightarrow \infty} \operatorname{det}\left(\tilde{M}_{n, l+1 \times l+1}^{\star}\right)=\lim _{n \rightarrow \infty} \prod_{i=1}^{l} \operatorname{det}\left(M_{n, i, i}^{\star}\right) \cdot \operatorname{det} C_{n, \mu \times \mu} \neq 0 .
$$

Notice that the limit is the same for every convergent subsequence and $\left\{\tilde{M}_{n, l+1 \times l+1}^{\star}\right\}$ is a bounded sequence. Thus, the above limit is true for the whole sequence. We conclude from (43) that (35) has a unique solution for $n$ large enough,

$$
\frac{h_{n}(z)}{\prod_{i=1}^{l}\left(z-\alpha_{i}\right)^{p_{i}} \pi(z) q_{n}(z)}
$$

which is the rational interpolation of type $(n, \mu+\nu)$ to $f(z)$ on the set $\Lambda(n+\mu+\nu)$. Furthermore, we have the estimations for the coefficients:

$$
\begin{aligned}
& \lim _{n \rightarrow \infty} n^{\tau} b_{k}^{(n)}=0 \quad \text { for } k=1,2, \ldots, \mu \text {, any } \tau, \\
& \lim _{n \rightarrow \infty} n^{p_{i}-k+1} a_{k, i}^{(n)}=\frac{d_{p_{i}}^{\{k\}} D_{p_{i}}^{\{k\}} H\left(\alpha_{i}\right)^{k-p_{i}-1}}{d_{p_{i}} D_{p_{i}}} \text { for } k=1,2, \ldots, p_{i}
\end{aligned}
$$

where $d_{p_{i}}$ and $D_{p_{i}}$ are constants only related to $p_{i}, H\left(\alpha_{i}\right)$, and $B_{r_{i}, i}$, and $d_{p_{i}}^{\{k\}}$ and $D_{p_{i}}^{\{k\}}$ are constants only related to $k, p_{i}, H\left(\alpha_{i}\right)$, and $B_{r_{i}, i} ; i=1, \ldots, l$. Thus (4) is 
true. Since $\nu=\sum_{i=1}^{l} p_{i}$, we have

$$
\begin{aligned}
& f(z)-R_{n, \mu+\nu}(z)=\frac{q_{n}(z) \pi(z) \prod_{i=1}^{l}\left(z-\alpha_{i}\right)^{p_{i}} f(z)-h_{n}(z)}{q_{n}(z) \pi(z) \prod_{i=1}^{l}\left(z-\alpha_{i}\right)^{p_{i}}} \\
& =\frac{1}{q_{n}(z) \pi(z) \prod_{i=1}^{l}\left(z-\alpha_{i}\right)^{p_{i}}} \\
& \times\left\{\sum_{l=1}^{\mu} b_{l}^{(n)}\left[\prod_{i=1}^{l}\left(z-\alpha_{i}\right)^{2 p_{i}} \pi_{l-1}(z) \pi(z) f(z)-w_{n, l}(z)\right]\right. \\
& +\sum_{i=1}^{l} \sum_{k=1}^{p_{i}} a_{k, i}^{(n)}\left[\left(z-\alpha_{i}\right)^{k-1+p_{i}} \prod_{j=1, j \neq i}^{l}\left(z-\alpha_{j}\right)^{2 p_{j}} \pi^{2}(z) f(z)-g_{n, k, 1}(z)\right] \\
& \left.+\prod_{i=1}^{l}\left(z-\alpha_{i}\right)^{2 p_{i}} \pi^{2}(z) f(z)-g_{n, p_{1}+1}(z)\right\} \\
& =\frac{\omega_{n+\mu+\nu}(z)}{q_{n}(z) \pi(z) \prod_{i=1}^{l}\left(z-\alpha_{i}\right)^{p_{i}}} \\
& \times\left\{\sum_{1 \leq i \leq l} \frac{C_{p_{i}, i} L_{p_{i}, i} n^{r_{i}-2 p_{i}-1}}{\left(z-\alpha_{i}\right) \omega_{n+\mu+\nu}\left(\alpha_{i}\right)}+\sum_{r_{i}-2 p_{i}-1=\lambda \geq 0} \frac{C_{p_{i}, i} n^{\lambda}}{\left(z-\alpha_{i}\right) \omega_{n+\mu+\nu}\left(\alpha_{i}\right)}+o(1)\right\} .
\end{aligned}
$$

Hence, (3) is true for $\lambda=\max _{1 \leq i \leq l}\left\{r_{i}-2 p_{i}-1\right\}=\max _{1 \leq i \leq m}\left\{r_{i}-2 p_{i}-1\right\}$.

Case 3. $v_{l}<\nu \leq v_{l+1}, m+1 \leq l \leq 2 m-1$. Set $p_{i}=p_{\nu, i}$ for $i=1, \ldots, m$. Then $p_{i}=r_{i}$ for $i=2 m-l+1, \ldots, m$, and (cf. [6])

$$
r_{i}-2 p_{i}-1 \leq r_{j}-2 p_{j} \leq r_{i}-2 p_{i}+1 \quad \text { for } 1 \leq i, j \leq 2 m-l .
$$

Construct the same $q_{n}(z)$ and $h_{n}(z)$ as in Case 2 for $l=m$. Similarly, we want $h_{n}(z)=\prod_{i=1}^{m}\left(z-\alpha_{i}\right)^{p_{i}} \pi(z) d_{n}(z)$, where $d_{n}(z)$ is a polynomial of degree $\leq n$, so we have

$$
\tilde{M}_{n, m+1 \times m+1} X_{n}=Y_{n}
$$

where $\tilde{M}_{n, m+1 \times m+1}, X_{n}$, and $Y_{n}$ are the same as in Case 2 for $l=m$. Furthermore,

$$
\begin{gathered}
\tilde{M}_{n, m+1 \times m+1}^{\star}:=\tilde{N}_{m+1 \times m+1} \cdot \tilde{M}_{n, m+1 \times m+1} \cdot \tilde{W}_{m+1 \times m+1}, \\
X_{n}^{\star}=\tilde{W}_{m+1 \times m+1}^{-1} \cdot X_{n}, \quad Y_{n}^{\star}=\tilde{N}_{m+1 \times m+1} \cdot Y_{n}
\end{gathered}
$$

satisfy

$$
\tilde{M}_{n, m+1 \times m+1}^{\star} X_{n}^{\star}=Y_{n}^{\star} .
$$

Here $\operatorname{det} C_{n, \mu \times \mu}$ still tends to a nonzero limit, and we still have

$$
\begin{aligned}
M_{j, m+1}^{\star}:=\lim _{n \rightarrow \infty} M_{n, j, m+1}^{\star}=V_{p_{j} \times \mu}, & j=1, \ldots, m, \\
M_{m+1, i}^{\star}:=\lim _{n \rightarrow \infty} M_{n, m+1, i}^{\star}=O_{\mu \times p_{i}}, & i=1, \ldots, m .
\end{aligned}
$$

We only need to study $M_{n, i, j}^{\star}$ now. It is easy to check by Definition 2 that

$$
r_{i}-2 p_{i} \leq 0 \quad \text { for } 1 \leq i \leq m .
$$

Thus, if $2 m-l+1 \leq j \leq m$, the $g_{n, k, j}(z)$ interpolates an analytic function. Furthermore, the interpolated function $\left(z-\alpha_{j}\right)^{k-1+p_{j}} \prod_{t=1, t \neq j}^{m}\left(z-\alpha_{t}\right)^{2 p_{t}} \pi(z) f(z)$ has a zero 
of order $k-1$ at $z=\alpha_{j}$ and a zero of order $2 p_{i}-r_{i}$ at $z=\alpha_{i}, i \neq j, 1 \leq i \leq m$. Hence, for elements of $M_{n, i, j}^{\star}$, (39) is true. This gives us

$$
\lim _{n \rightarrow \infty} M_{n, i, j}^{\star}=O_{p_{i} \times p_{j}} \quad \text { for } \quad 2 m-l+1 \leq j \leq m, 1 \leq i \leq m, i \neq j .
$$

As for a fixed $j \leq 2 m-l, M_{n, i, j}^{\star}$ may not tend to a zero matrix, but they are bounded. In fact, $g_{n, k, j}^{(s)}(z)$ interpolates an analytic function when $r_{j}-p_{j}-k+1 \leq 0$. For this situation, the above reasoning is still valid. That means

$$
\begin{gathered}
\lim _{n \rightarrow \infty} n^{-\left(r_{i}-2 p_{i}+p_{j}-k+1+s\right)} g_{n, k, j}^{(s)}\left(\alpha_{i}\right)=0, \\
0 \leq s \leq p_{j}-1, \quad 1 \leq i \leq m, \quad i \neq j, \quad k \geq r_{j}-p_{j}+1 .
\end{gathered}
$$

When $1 \leq k<r_{j}-p_{j}+1$, the interpolated function

$$
\left(z-\alpha_{j}\right)^{k-1+p_{j}} \prod_{t=1, t \neq j}^{m}\left(z-\alpha_{t}\right)^{2 p_{t}} \pi(z) f(z)
$$

has only one pole at $z=\alpha_{j}$ of order $r_{j}-p_{j}-k+1$, and (23) and (24) are true here. For $1 \leq i \leq 2 m-l,(45)$ is true; for $2 m-l<i \leq m$,

$$
\begin{aligned}
r_{j}-2 p_{j}-1 & \leq r_{j}-2\left[k_{l}+u_{j, 2 m-l}+r_{2 m-l+1}\right]-1 \\
& \leq r_{j}-2 k_{l}-\left(r_{j}-r_{2 m-l+1}-1\right)-2 r_{2 m-l+1}-1 \\
& =-2 k_{l}-r_{2 m-l+1} \leq-r_{2 m-l+1} \leq-r_{i}=r_{i}-2 p_{i} .
\end{aligned}
$$

Hence,

$$
\begin{aligned}
\lim \sup _{n \rightarrow \infty} n^{-\left(r_{i}-2 p_{i}+p_{j}-k+1+s\right)}\left|g_{n, k, j}^{(s)}\left(\alpha_{i}\right)\right| \\
\quad=\limsup _{n \rightarrow \infty} n^{-\left(r_{i}-2 p_{i}-r_{j}+2 p_{j}+1\right)} n^{-\left(r_{j}-p_{j}-k+s\right)}\left|g_{n, k, j}^{(s)}\left(\alpha_{i}\right)\right|<\infty,
\end{aligned}
$$

for $0 \leq s \leq p_{j}-1, \quad 1 \leq i \leq m, \quad i \neq j, \quad k<r_{j}-p_{j}+1$. We conclude that for any convergent subsequence,

$$
\begin{aligned}
\lim _{n \rightarrow \infty} \operatorname{det}\left(\tilde{M}_{n, m+1 \times m+1}^{\star}\right)=\lim _{n \rightarrow \infty} \operatorname{det}\left(\begin{array}{ccc}
M_{n, 1,1}^{\star} & \cdots & M_{n, 1,2 m-l}^{\star} \\
\vdots & & \vdots \\
M_{n, 2 m-l, 1}^{\star} & \cdots & M_{n, 2 m-l, 2 m-l}^{\star}
\end{array}\right) \\
\quad \times \operatorname{det}\left(\begin{array}{cccc}
M_{n, 2 m-l+1,2 m-l+1}^{\star} & \ddots & & \\
& & M_{n, m, m}^{\star} & \\
& & & C_{n, \mu \times \mu}
\end{array}\right) .
\end{aligned}
$$

Applying reasoning similar to that in Case 2 to the matrix in the first determinant, we deduce that (42) is still true. For the second determinant,

$$
\lim _{n \rightarrow \infty} n^{-\left(r_{j}-p_{j}-k+1+s\right)} g_{n, k, j}^{(s)}\left(\alpha_{j}\right)=n^{-(-k+1+s)} g_{n, k, j}^{(s)}\left(\alpha_{j}\right)= \begin{cases}B_{r_{j}, j} & \text { when } k=s+1, \\ 0 & \text { when } k \neq s+1\end{cases}
$$

Thus, (37) still holds here. We come to the conclusion

$$
\begin{aligned}
\operatorname{det}\left(\tilde{M}_{m+1 \times m+1}^{\star}\right) & =\lim _{n \rightarrow \infty} \operatorname{det}\left(\tilde{M}_{n, m+1 \times m+1}^{\star}\right) \\
& =\lim _{n \rightarrow \infty} \prod_{i=1}^{m} \operatorname{det}\left(M_{n, i, i}^{\star}\right) \cdot \operatorname{det} C_{n, \mu \times \mu} \neq 0 .
\end{aligned}
$$

Thus, all conclusions in case 2 are still valid for this case. This means that (46) has a unique solution for $n$ large enough, $h_{n}(z) /\left(\prod_{i=1}^{m}\left(z-\alpha_{i}\right)^{p_{i}} \pi(z) q_{n}(z)\right)$, which is the 
rational interpolation of type $(n, \mu+\nu)$ to $f(z)$ on the set $\Lambda(n+\mu+\nu)$. The estimates (44) for the coefficients are still true. Since $\nu=\sum_{i=1}^{m} p_{i}$, we have

$$
\begin{aligned}
& f(z)-R_{n, \mu+\nu}(z)=\frac{\omega_{n+\mu+\nu}(z)}{q_{n}(z) \pi(z) \prod_{i=1}^{m}\left(z-\alpha_{i}\right)^{p_{i}}} \\
& \quad \times\left\{\sum_{1 \leq i<2 m-l} \frac{C_{p_{i}, i} L_{p_{i}, i} n^{r_{i}-2 p_{i}-1}}{\left(z-\alpha_{i}\right) \omega_{n+\mu+\nu}\left(\alpha_{i}\right)}+\sum_{r_{i}-2 p_{i}-1=\lambda \geq 0} \frac{C_{p_{i}, i} n^{\lambda}}{\left(z-\alpha_{i}\right) \omega_{n+\mu+\nu}\left(\alpha_{i}\right)}+o(1)\right\} .
\end{aligned}
$$

Hence, (3) is valid for $\lambda=\max _{1 \leq i \leq m}\left\{r_{i}-2 p_{i}-1\right\}$.

The proof of Theorem 1 is completed.

\section{Other results}

Corollary 1. Let $f(z)$ be as in Theorem 1. Suppose that $\nu$ is good by Definition 1, and $R_{n, \mu+\nu}(z)$ is a rational function of type $(n, \mu+\nu)$ interpolating $f(z)$ on the set $\Lambda(n+\mu+\nu)$. Then, except for a finite number of points, the inequality

$$
\limsup _{n \rightarrow \infty}\left|\frac{R_{n, \mu+\nu}(z) \rho^{n}}{n^{\lambda} \exp (n G(z))}\right|>0
$$

holds for each $z$ exterior to $\Gamma_{\rho}$. Consequently, the sequence $\left\{R_{n, \mu+\nu}(z)\right\}_{n=0}^{\infty}$ can converge in at most a finite number of points exterior to $\Gamma_{\rho}$.

Proof. Assume $\nu$ is good; according to the proof of Theorem 1, we get

$$
f(z)-R_{n, \mu+\nu}(z)=\frac{\omega_{n+\mu+\nu}(z)}{q_{n}(z) \pi(z) \prod_{i=1}^{m}\left(z-\alpha_{i}\right)^{p_{i}}}\left\{\sum_{i=1}^{m} \frac{C_{\nu, i} n^{r_{i}-2 p_{i}-1}}{\left(z-\alpha_{i}\right) \omega_{n+\mu+\nu}\left(\alpha_{i}\right)}+o(1)\right\},
$$

where, if $\nu<\sum_{i=1}^{m} r_{i}$, then at least one $C_{\nu, i} \neq 0, i=1,2, \ldots, m$. Thus,

$$
\left|\left[f(z)-R_{n, \mu+\nu}(z)\right] q_{n}(z) \frac{\omega_{n+\mu+\nu}\left(\alpha_{1}\right)}{\omega_{n+\mu+\nu}(z) n^{\lambda}}\right| \leq A
$$

for any compact set exterior to $\Gamma_{\rho}$. We conclude that the functions inside the absolute value form a normal family. Consequently, there exists a convergent subsequence. Because (for at least a subsequence)

$$
\lim _{n \rightarrow \infty} \sum_{i=1}^{m} \frac{C_{\nu, i} n^{r_{i}-2 p_{i}-1-\lambda} \omega_{n+\mu+\nu}\left(\alpha_{1}\right)}{\left(z-\alpha_{i}\right) \omega_{n+\mu+\nu}\left(\alpha_{i}\right)}=C(z) \not \equiv 0
$$

and

$$
\lim _{n \rightarrow \infty} f(z) q_{n}(z) \frac{\omega_{n+\mu+\nu}\left(\alpha_{1}\right)}{\omega_{n+\mu+\nu}(z) n^{\lambda}}=0
$$

for $z$ exterior to $\Gamma_{\rho}$, we obtain

$$
\limsup _{n \rightarrow \infty}\left|R_{n, \mu+\nu}(z) \rho^{n} \exp (-n G(z)) n^{-\lambda}\right|>0 \quad \text { except in a finite set. }
$$

This means that $\left\{R_{n, \mu+\nu}(z)\right\}_{n=0}^{\infty}$ is divergent in $\mathbf{C} \backslash E_{\rho} \cup \Gamma_{\rho}$ except at finite points.

Remark. If we let $\beta_{i}^{(n)}=0$ for $1 \leq i \leq n+1$, all $n$, then $\omega_{n}(z)=z^{n+1}, E=$ $\{z:|z| \leq \delta\}$ satisfy the assumptions and the multipoint Padé approximations become Padé approximations. Hence, our results also can be applied to Padé approximations. 


\section{An example for "bad" $\nu$ 's}

In this section, we give a proposition about "bad" $\nu$ 's. The investigation on the poles of $\{[n / 1](z)\}_{n=0}^{\infty}$ for the function $f(z)$ with exactly two different simple poles (cf. [1], p.239) has been done. We point out that the limit point set of the poles could make up a continuous curve.

Proposition 1. Let $E=\{z:|z| \leq 1 / 2\}, E_{\rho}$ be the open unit disk, and

$$
f(z)=\frac{g(z)}{(z-a)(z-b)}
$$

where $|a|=|b|=1$ and $a \neq b$, and $g(z)=\sum_{n=0}^{\infty} g_{n} z^{n}$ is analytic on $|z| \leq 1$ with $g(a) \neq 0$ and $g(b) \neq 0$. Let $\phi=\arg (b / a)$ and $[n / 1](z)$ be the $(n, 1)$ Padé approximant of $f(z)$. Then, if $\phi / \pi$ is a rational number $t / s$ ( $t$ and $s$ have no common factors), the sequence $\{[n / 1](z)\}$ has at most $2 s$ distinct poles; if $\phi / \pi$ is irrational, the limit point set of poles of $\{[n / 1](z)\}$ make up an arc of a circle in $|z|<1$. Let $O$ be the closure of the set of poles of $\{[n / 1](z)\}$, then $\{[n / 1](z)\}$ is uniformly convergent on any compact subset of $E_{\rho} \backslash O$.

Proof. The function $f(z)$ is analytic on $E_{\rho}$ and analytic on $\Gamma_{\rho}$ except at 2 points $z_{1}=a, z_{2}=b$. The function $f(z)$ and $E$ satisfy the assumptions, so we can apply Definitions 1 and 2. Since $m=2, r_{1}=1, r_{2}=1$, good values of $\nu$ are 0 and 2. The value $\nu=1$ is "bad". Now, let us calculate the Padé approximants $\{[n / 1](z)\}$ and their poles.

We consider first $g(z)=1$. Because the Taylor expansion of $f(z)$ is

$$
f(z)=\sum_{n=0}^{\infty} f_{n} z^{n}=\sum_{n=0}^{\infty} \frac{1}{(b-a)}\left(\frac{1}{a^{n+1}}-\frac{1}{b^{n+1}}\right) z^{n}
$$

for each $n$, with $f_{-1}:=0$,

$$
\begin{aligned}
{[n / 1](z) } & =\frac{\sum_{j=0}^{n} z^{j} \operatorname{det}\left(\begin{array}{cc}
f_{j} & f_{j-1} \\
f_{n+1} & f_{n}
\end{array}\right)}{\operatorname{det}\left(\begin{array}{cc}
1 & z \\
f_{n+1} & f_{n}
\end{array}\right)}=\frac{\sum_{j=0}^{n}\left(-f_{n+1} f_{j-1}+f_{n} f_{j}\right) z^{j}}{-f_{n+1} z+f_{n}} \\
& =\frac{\left(-f_{n+1} z+f_{n}\right) \sum_{j=0}^{n-1} f_{j} z^{j}+f_{n}^{2} z^{n}}{-f_{n+1} z+f_{n}} .
\end{aligned}
$$

When $f_{n} \neq 0$, it is a non-degenerate case. Furthermore, when $f_{n+1}=0$, there is no pole; when $f_{n+1} \neq 0$, the pole of $[n / 1](z)$ should be

$$
\begin{aligned}
z_{n, p} & =\frac{f_{n}}{f_{n+1}}=\frac{\frac{1}{b-a}\left(\frac{1}{a^{n+1}}-\frac{1}{b^{n+1}}\right)}{\frac{1}{b-a}\left(\frac{1}{a^{n+2}}-\frac{1}{b^{n+2}}\right)} \\
& =\frac{a\left(1-e^{i \phi} e^{-(n+2) i \phi}\right)}{1-e^{-(n+2) i \phi}}
\end{aligned}
$$

where $e^{-i \phi}:=a / b$. If $\phi / \pi$ is rational, then there will be finite distinct poles $\left\{z_{n, p}\right\}$. For the special case $\phi=\pi$, the Padé approximant $[n / 1](z)$ is either a degenerate case or has a constant denominator, so there is no pole. On the other hand, if $\phi / \pi$ is irrational, by a result due to Bohl, Sierpinski, and Weyl (cf. [10]), $\left\{e^{i k \phi}\right\}_{k=0}^{\infty}$ is uniformly distributed on the unit circle, i.e., the sequence is dense on the unit circle. 
Thus, if $\phi / \pi$ is irrational, then the limit point set of $\left\{z_{n, p}\right\}$ consists of points of the form

$$
z_{\theta}=\frac{a\left(1-e^{i \phi} e^{i \theta}\right)}{1-e^{i \theta}}, \quad \theta \in[0,2 \pi]
$$

Generally, if

$$
f(z)=\frac{g(z)}{(z-a)(z-b)}
$$

where $g(z)=\sum_{n=0}^{\infty} g_{n} z^{n}$ is analytic on $|z| \leq 1$ and $g(a) \neq 0, g(b) \neq 0$, similar to before, then for the non-degenerate case and $f_{n+1} \neq 0$, the pole of the Padé approximant $[n / 1](z)$ of $f(z)$ is

$$
\begin{aligned}
z_{n, p}=\frac{f_{n}}{f_{n+1}} & =\frac{\sum_{k=0}^{n} \frac{1}{b-a}\left(\frac{1}{a^{n+1-k}}-\frac{1}{b^{n+1-k}}\right) g_{k}}{\sum_{k=0}^{n+1} \frac{1}{b-a}\left(\frac{1}{a^{n+2-k}}-\frac{1}{b^{n+2-k}}\right) g_{k}} \\
& =\frac{a^{-n-1} g(a)+\epsilon_{n}-b^{-n-1} g(b)-\varepsilon_{n}}{a^{-n-2} g(a)+\epsilon_{n+1}-b^{-n-2} g(b)-\varepsilon_{n+1}} \\
& =\frac{a\left(r-e^{i \phi} e^{-(n+2) i \phi}\right)+a^{n+2}\left(\epsilon_{n}-\varepsilon_{n}\right) / g(b)}{r-e^{-(n+2) i \phi}+a^{n+2}\left(\epsilon_{n+1}-\varepsilon_{n+1}\right) / g(b)}
\end{aligned}
$$

where $r:=g(a) / g(b)($ recall $g(b) \neq 0)$ and

$$
\begin{aligned}
& \epsilon_{n}:=a^{-n-1}\left(\sum_{k=0}^{n} g_{k} a^{k}-g(a)\right) \rightarrow 0, \\
& \varepsilon_{n}:=b^{-n-1}\left(\sum_{k=0}^{n} g_{k} b^{k}-g(b)\right) \rightarrow \infty
\end{aligned}
$$

as $n \rightarrow 0$, since $|a|=1,|b|=1$. For convergent subsequences,

$$
z_{n_{j}, p} \rightarrow \frac{a\left(r-e^{i \phi} e^{i \theta}\right)}{r-e^{i \theta}} \text { for } \quad n_{j} \rightarrow \infty \text { and some } \theta \in[0,2 \pi) .
$$

Notice that if $|r| \neq 1$, then $f_{n} \neq 0$ for $n$ large enough; thus $[n / 1](z)$ is a non-degenerate case for $n$ large enough. If $r=e^{i \gamma}$ for some $\gamma \in[0,2 \pi)$, then the only degenerate cases are in the subsequence $\left\{\left[n_{j} / 1\right](z)\right\}$ for $n_{j}$ 's satisfying $e^{i \gamma}=e^{-\left(n_{j}+1\right) i \phi}$. For $n_{j}$ 's satisfying $e^{i \gamma}=e^{-\left(n_{j}+2\right) i \phi}$, the subsequence $\left\{\left[n_{j} / 1\right](z)\right\}$ has no poles because all denominators are constants.

For the same reason, if $\phi / \pi$ is rational, then there only will be finite distinct poles $\left\{z_{n, p}\right\}$. Obviously, if we write $\phi / \pi:=t / s$ where $t$ and $s$ are integers having no common factors, then there will be at most $2 s$ distinct poles. On the other hand, if $\phi / \pi$ is irrational, then the limit point set of $\left\{z_{n, p}\right\}$ is made up of points of the form

$$
z_{\theta}=\frac{a\left(r-e^{i \phi} e^{i \theta}\right)}{r-e^{i \theta}}, \quad \theta \in[0,2 \pi] \text {. }
$$

Since $w=\frac{a\left(r-e^{i \phi} z\right)}{r-z}$ is a Möbius transformation on the complex plane, it maps a circle not passing through its pole to a circle and a circle passing through its pole to a straight line. For our problem, the circle being mapped is the unit circle, so if $|r|=1$, the image set or point set $\left\{\left(x(\theta)=\operatorname{Re}\left(z_{\theta}\right), y(\theta)=\operatorname{Im}\left(z_{\theta}\right)\right)\right\}$ is a straight line passing 
through zero. For $|r| \neq 1$, since we can find a $\theta$ such that $\left|z_{\theta}\right|<1,\left(e^{i \phi} \neq 1\right)$, a part of the circle $\left\{\left(x(\theta)=\operatorname{Re}\left(z_{\theta}\right), y(\theta)=\operatorname{Im}\left(z_{\theta}\right)\right)\right\}$, or an arc, is inside $|z|<1$. Now

$$
O=\left\{z:|z|<1 ; z=\frac{a\left(r-e^{i \phi} e^{i \theta}\right)}{r-e^{i \theta}} \quad \text { for } 0 \leq \theta \leq 2 \pi\right\}
$$

is the closure of the set of poles of $\{[n / 1](z)\}_{n=0}^{\infty}$. Because the Padé sequence $\{[n / 1]$ $(z)\}_{n=0}^{\infty}$ converges on $|z|<1$ except on the limit points of the poles, our proposition is true.

\section{An example of applications}

One application of our results is to Szegö polynomials associated with Wiener-Levinson filters [3].

Definition 3. Doubly-infinite sequences $x=\{x(m)\}_{m=-\infty}^{\infty}$ of real numbers are called (discrete) signals. Here we consider $N$-truncated causal signals $x_{N}=\left\{x_{N}(m)\right\}$ since we can get only finite data, where

$$
x_{N}(m):= \begin{cases}x(m), & \text { if } 0 \leq m \leq N-1 \\ 0, & \text { otherwise }\end{cases}
$$

and $x_{N}(0) \neq 0$. The $Z$-transform of $x_{N}$ is given by $X_{N}(z):=\sum_{m=0}^{N-1} x_{N}(m) z^{-m}$.

For the absolutely continuous distribution function $\phi_{N}(\theta)$ given by

$$
\phi_{N}^{\prime}(\theta):=\frac{1}{2 \pi}\left|X_{N}\left(e^{i \theta}\right)\right|^{2}, \quad-\pi \leq \theta \leq \pi,
$$

the inner product is defined by

$$
\langle f, g\rangle:=\int_{-\pi}^{\pi} f\left(e^{i \theta}\right) \overline{g\left(e^{i \theta}\right)} d \phi_{N}(\theta), \quad f, g \in \mathbf{C}_{0} .
$$

We denote by $\left\{\rho_{n}(z)=\rho_{n}\left(\phi_{N} ; z\right)\right\}_{n=0}^{\infty}$ the sequence of monic Szegö polynomials orthogonal with respect to $\phi_{N}$, and denote the reciprocal polynomials by

$$
\rho_{n}^{*}(z):=\rho_{n}^{*}\left(\phi_{N} ; z\right):=z^{n} \overline{\rho_{n}\left(\phi_{N} ; 1 / \bar{z}\right)}, \quad n=0,1,2, \ldots .
$$

With these definitions, we have a theorem by Jones et al. [3].

Theorem B. Let

$$
D_{N}(z)= \pm x_{N}(0) \prod_{\left|z_{k}\right| \geq 1}\left(z-z_{k}\right) \prod_{\left|z_{k}\right|<1}\left(1-\bar{z}_{k} z\right)
$$

where the $z_{k}^{\prime} s$ are all zeros of the polynomial $z^{N-1} X_{N}(z)$, and the sign is chosen so that $D_{N}(0)>0$. Define $F_{N}(z):=1 / D_{N}(z) D_{N}^{*}(z)$. Let $M$ denote the degree of $D_{N}(z)$, $0 \leq M \leq N-1$, and let $[n / M]$ be the classical Padé approximant of type $(n, M)$ to the rational function $F_{N}(z)$. Then there exists a polynomial $Q_{n, M}(z)$ (of degree $\left.\leq M\right)$ such that

$$
[n / M](z)=\frac{\rho_{n}^{*}(z)}{Q_{n, M}(z)}
$$

Now we can use our theorem for this case. Because all poles of $F_{N}(z)$ on $|z|=1$ have even degrees, $r_{1}, r_{2}, \ldots, r_{m}$ in Theorem 1 are all even numbers. If the number of poles of $F_{N}(z)$ in $|z|<1$ is $\mu$, then the number of poles of $F_{N}(z)$ on $|z|=1$ is $2(M-\mu)$. By our Definitions 1 and 2, we can verify that $\nu:=M-\mu$ is good. Thus, we have a corollary. 
Corollary 2. With the hypotheses of Theorem B,

$$
\lim _{n \rightarrow \infty}[n / M](z)=\lim _{n \rightarrow \infty} \frac{\rho_{n}^{*}(z)}{Q_{n, M}(z)}=\frac{1}{D_{N}(z) D_{N}^{*}(z)}
$$

locally uniformly on the set $\{|z|<1\} \backslash\left\{\right.$ zeros of $\left.D_{N}^{*}(z)\right\}$. Moreover, the degree of $Q_{n, M}(z)$ is exactly $M$, and the $M$ zeros of $Q_{n, M}(z)$ approach the $M$ zeros of $D_{N}^{*}(z)$.

Next, let us study the limit function outside $\{z:|z|<1\}$ when $D(z)$ has at least one zero on $|z|=1$. Note here that $E=\{z:|z| \leq 1\}$ and $H(z)=-z^{-1}$. Write $D_{N}^{*}(z)=a_{\mu} \pi(z) \prod_{i=1}^{m}\left(z-\alpha_{i}\right)^{s_{i}}$ where $\pi(z)$ is a polynomial with all zeros inside $|z|<1$ and $\left\{\alpha_{i}\right\}_{i=1}^{m}$ are all on $|z|=1$. Let $b_{\mu}=\bar{a}_{\mu} \prod_{i=1}^{m}\left(-\bar{\alpha}_{i}\right)^{s_{i}}$; then by calculating all constants in (31), we have for $z \bar{\epsilon}\left\{\alpha_{1}, \alpha_{2}, \ldots, \alpha_{m}\right\}$,

$$
\rho_{n}^{*}(z)=\frac{Q_{n, M}(z)}{D_{N}(z) D_{N}^{*}(z)}+\frac{z^{n+M+1}}{c b_{\mu} D_{N}^{*}(z) n} \sum_{i=1}^{m} \frac{\pi\left(\alpha_{i}\right)(-1)^{s_{i}} \alpha_{i} s_{i}}{\pi^{*}\left(\alpha_{i}\right)\left(z-\alpha_{i}\right) \alpha_{i}^{n+M+1}}+o\left(n^{-1}\right) .
$$

From this estimate, we see that the Padé approximant sequence is divergent outside $E$. Because we are interested in the asymptotic behavior of Szegö orthogonal polynomials, we need the following estimate for $\rho_{n}(z)$ if $z \bar{\epsilon}\left\{\alpha_{1}, \alpha_{2}, \ldots, \alpha_{m}\right\}$,

$$
\rho_{n}(z)=\frac{z^{n+M} Q_{n, M}^{*}(z)}{D_{N}^{*}(z) D_{N}(z)}+\frac{1}{\bar{c} \bar{b}_{\mu} D_{N}(z) n} \sum_{i=1}^{m} \frac{\pi^{*}\left(\alpha_{i}\right)(-1)^{s_{i}-1} s_{i} \alpha_{i}^{n+M+1}}{\pi\left(\alpha_{i}\right)\left(z-\alpha_{i}\right)}+o\left(n^{-1}\right) .
$$

With this asymptotic formula, we can study some interesting behavior of $\rho_{n}(z)$. We will focus the rest of this section on a discussion of the zero distribution of $\left\{\rho_{n}(z)\right\}_{n=1}^{\infty}$. We can get the same theorems as Szabados [9]. However, our proof is much simpler. We will use the notation $Z\left(F_{N}\right)$ for the set of cluster-points of the roots of the $\rho_{n}(z)$ 's.

\section{Proposition 2.}

$$
Z\left(F_{N}\right) \bigcap\{z:|z|>1\}=\emptyset
$$

Proof. By (50), we get

$$
z^{-n} \rho_{n}(z) \rightarrow \frac{z^{M} D_{N}^{*}(z)}{D_{N}(z) D_{N}^{*}(z)} \quad \text { for }|z|>1 \quad \text { as } n \rightarrow \infty \text {. }
$$

The function $z^{M} / D_{N}(z)$ has no zero in $|z| \geq 1+\varepsilon$ for any $\varepsilon>0$. Thus, by Hurwitz's theorem, $z^{-n} \rho_{n}(z)$ has no zeros in $|z| \geq 1+\varepsilon$ for $n$ large enough.

We need a deep and general theorem of Erdös and Turan [2] for the proof of next proposition.

Theorem C. If $\xi_{1}, \xi_{2}, \ldots, \xi_{N}$ are roots of the polynomial $a_{0}+a_{1} z+\cdots+a_{N} z^{N}$ $\left(a_{0}, a_{N} \neq 0\right)$, then for any $0 \leq \alpha \leq \beta \leq 2 \pi$, we have

$$
\left|\sum_{\alpha \leq \arg \xi \leq \beta} 1-N(\beta-\alpha) / 2 \pi\right| \leq 16 \sqrt{N \log \left(\sum_{j=0}^{N}\left|a_{j}\right| / \sqrt{\left|a_{0}\right|\left|a_{N}\right|}\right)} .
$$

\section{Proposition 3.}

$$
\{z:|z|=1\} \subset Z\left(F_{N}\right) .
$$

Proof. By the same reasoning as in the proof of Theorem 4 in [9], we know that for any $\varepsilon>0$, there exists a subsequence $\left\{n_{j}\right\}_{j=0}^{\infty}$ of integers such that for some $p, 0 \leq p<m$,

$$
\lim _{j \rightarrow \infty} \alpha_{l}^{n_{j}}=\alpha_{l}^{p-M-1} \quad \text { for } l=1,2, \ldots, m
$$


Hence (50) implies

$$
\lim _{j \rightarrow \infty} n_{j} \rho_{n_{j}}(z) D_{N}(z)=\frac{1}{\bar{c} \bar{b}_{\mu}} \sum_{i=1}^{m} \frac{\pi^{*}\left(\alpha_{i}\right)(-1)^{s_{i}-1} s_{i} \alpha_{i}^{p}}{\pi\left(\alpha_{i}\right)\left(z-\alpha_{i}\right)} \quad \text { for } \quad|z| \leq 1-\varepsilon .
$$

By Hurwitz's theorem, this limit means that for $j$ sufficiently large $\left(j \geq j_{1}(\varepsilon)\right)$, the polynomial $\rho_{n_{j}}(z)$ has at most $m-1$ zeros in $|z| \leq 1-\varepsilon$. On the other hand, by Proposition 3 , for sufficiently large $j\left(j \geq j_{2}(\varepsilon)\right), \rho_{n_{j}}(z)$ does not have zeros in $|z| \geq 1-\varepsilon$. Hence, $\rho_{n_{j}}(z)$ has at least $n_{j}-m+1$ roots in $1-\varepsilon<|z|<1+\varepsilon$ provided that $j \geq j_{3}(\varepsilon):=\max \left\{j_{1}(\varepsilon), j_{2}(\varepsilon)\right\}$.

The next step will be to prove that $n_{j}-m+1$ roots of $\rho_{n_{j}}(z)$ are asymptotically uniformly distributed in angles with vertex at 0 . Here we will apply Theorem $C$. Hence, we need to calculate $\sum_{j=0}^{N}\left|a_{j}\right| / \sqrt{\left|a_{0}\right|\left|a_{N}\right|}$ for $\rho_{n}(z)$. Obviously, the value is the same for $\rho_{n}(z)$ and $\rho_{n}^{*}(z)$. Let $h_{n}(z)=c \prod_{i=1}^{m}\left(z-\alpha_{i}\right)^{s_{i}} \pi(z) \rho_{n}^{*}(z)$. Since $\left|\alpha_{i}\right|=1, \sum_{j=0}^{N}\left|a_{j}\right|$ is bounded by $(n+M)^{M}$ multiplying the sum of the absolute value of coefficients of $h_{n}(z)$.

From the proof of Theorem 1, we have that

$$
\begin{aligned}
h_{n}(z) & =\sum_{l=1}^{\mu} b_{l}^{(n)} w_{n, l}(z)+\sum_{i=1}^{m} \sum_{k=1}^{s_{i}} a_{k, i}^{(n)} g_{n, k, i}(z)+g_{n, s_{1}+1}(z) \\
& =: \sum_{l=1}^{\mu} b_{l}^{(n)} \sum_{j=0}^{n+M} d_{j}^{(l)} z^{j}+\sum_{i=1}^{m} \sum_{k=1}^{s_{i}} a_{k, i}^{(n)} \sum_{j=0}^{n+M} e_{j, i}^{(k)} z^{j}+\sum_{j=0}^{n+M} e_{j, s_{1}+1} z^{j}=: \sum_{i=0}^{n+M} h_{i}^{(n)} z^{i}
\end{aligned}
$$

where the $b_{l}^{(n)}$ 's and $a_{k, i}^{(n)}$ 's satisfy (44). Furthermore, $w_{n, l}(z)$ and $g_{n, k, i}(z)$ are $n+M$ th truncated Maclaurin's series of

$$
\pi_{l-1}(z) \pi(z) \prod_{i=1}^{m}\left(z-\alpha_{i}\right)^{2 s_{i}} F_{N}(z)
$$

and

$$
\pi^{2}(z)\left(z-\alpha_{i}\right)^{k-1+s_{i}} \prod_{j=1, j \neq i}^{m}\left(z-\alpha_{j}\right)^{2 s_{j}} F_{N}(z)
$$

respectively. Since $\pi_{l-1}(z) \pi(z) \prod_{i=1}^{m}\left(z-\alpha_{i}\right)^{2 s_{i}} F_{N}(z)$ is analytic in $|z|<\sigma$ and $\sigma>1$, $\left|d_{j}^{(l)}\right|$ are bounded for all $j$ and $l$. Thus, because of (44), for any $1>\delta>0$,

$$
\left|\sum_{l=1}^{\mu} b_{l}^{(n)} d_{j}^{(l)}\right|<\delta /(n+1) \quad \text { for } n \text { large enough, } 1 \leq j \leq n+M .
$$

For the second sum, let

$$
\begin{aligned}
\pi^{2}(z)\left(z-\alpha_{i}\right)^{k-1+s_{i}} & \prod_{j=1, j \neq i}^{m}\left(z-\alpha_{j}\right)^{2 s_{j}} F_{N}(z) \\
= & \frac{\pi(z)}{a_{\mu} b_{\mu} \pi^{*}(z)} \frac{1}{\left(z-\alpha_{i}\right)^{k-1+s_{i}} \prod_{j=1, j \neq i}^{m}\left(z-\alpha_{j}\right)^{s_{j}}}=: \sum_{j=0}^{\infty} e_{j, i}^{(k)} z^{j} .
\end{aligned}
$$

It is obvious that for some constant $C$,

$$
\left|e_{j, i}^{(k)}\right| \leq C\left(j+s_{i}\right)^{s_{i}-k+1} \leq C\left(n+s_{i}\right)^{s_{i}-k+1} \quad \text { for } 0 \leq j \leq n .
$$


According to (53) and (54), for $n$ large enough and a constant $L$,

$$
\begin{aligned}
\sum_{j=0}^{n}\left|h_{j}^{(n)}\right| & \leq \sum_{l=1}^{\mu}\left|b_{l}^{(n)} d_{j}^{(l)}\right|+\sum_{i=1}^{m} \sum_{k=1}^{s_{i}}\left|a_{k, i}^{(n)} e_{j, i}^{(k)}\right| \\
& \leq \delta+\sum_{i=1}^{m} \sum_{k=1}^{s_{i}}\left|a_{k, i}^{(n)}\right| C\left(n+s_{i}\right)^{s_{i}-k+1} \leq L .
\end{aligned}
$$

We are almost finished except for finding a lower bound for $h_{n+M}^{(n)}$. From (52), we see that there is a subsequence $\left\{n_{j}\right\}_{j=1}^{\infty}$ and an integer $p$ such that

$$
\lim _{j \rightarrow \infty} h_{n_{j}+M}^{\left(n_{j}\right)}=\lim _{j \rightarrow \infty} n_{j} \rho_{n_{j}}(0) D_{N}(0)=\frac{1}{\bar{c} \bar{b}_{\mu}} \sum_{i=1}^{m} \frac{\pi^{*}\left(\alpha_{i}\right)(-1)^{s_{i}} s_{i} \alpha_{i}^{p}}{\pi\left(\alpha_{i}\right)} \neq 0 .
$$

As for $h_{0}^{(n)}$, it goes to a non-zero constant $D_{N}^{*}(0) / D_{N}(0)$. Since $h_{n+M}^{(n)}=c a_{n}$ and $h_{0}^{(n)}=c \pi(0)(-\alpha)^{s} a_{0}$, combining these with (55), we obtain, for $n$ large enough (at least for a subsequence $\left.\left\{n_{j}\right\}_{j=1}^{\infty}\right)$,

$$
\log \left(\sum_{j=0}^{n}\left|a_{j}\right| / \sqrt{\left|a_{0}\right|\left|a_{n}\right|}\right) \leq \log (n+M)^{(M+1)}=(M+1) \log (n+M) .
$$

Let any $\xi_{0} \in|z|=1, \theta_{0}=\arg \xi_{0}$, and choose $N$ such that

$$
16 \sqrt{M+1} \sqrt{n \log (n+M)}<n \varepsilon /(2 \pi) \quad \text { whenever } n>N(\varepsilon) .
$$

Then

$$
\begin{aligned}
\frac{n 2 \varepsilon}{(2 \pi)}-16 \sqrt{M+1} \sqrt{n \log (n+M)} & \leq \sum_{\left|\arg z-\theta_{0}\right| \leq \varepsilon} 1 \\
& \leq \frac{n 2 \varepsilon}{(2 \pi)}+16 \sqrt{M+1} \sqrt{n \log (n+M)} .
\end{aligned}
$$

Hence, if we let $j \geq N$, then $\rho_{n_{j}}^{*}(z)$ has at least $n_{j} \varepsilon /(2 \pi)-m+1$ roots in the " $\varepsilon$ neighborhood" of the point $\xi_{0}:\left\{z: 1-\varepsilon<|z|<1+\varepsilon,\left|\arg z-\theta_{0}\right| \leq \varepsilon\right\}$, which means that $\xi_{0} \in Z\left(F_{N}\right)$. By the arbitrariness of $\xi_{0} \in|z|=1$, we conclude that $\{z:|z|=1\} \subset Z\left(F_{N}\right)$.

Acknowledgment. The research of this author was done in partial fulfillment of the Ph.D. degree at the University of South Florida under the direction of Prof. E. B. Saff.

\section{References}

1. G. A. Baker and P. Graves-Morris, Padé Approximation, Part I: Basic Theory, Encyclopedia of Mathematics and Its Applications, Vol. 13, Addison-Wesley, Reading, Mass. (1981).

2. P. Erdös and P. Turán, Distribution of roots of polynomials, Ann. of Math. 5 (1950), 105-119.

3. W. B. Jones, O. Njastad, and E. B. Saff, Szegö polynomials associated with Wiener-Levinson filters, J. Comp. Appl. Math. 32 (1990), 387-406.

4. R. de Montessus de Ballore, Sur les functions continus algebriques, Bull. Soc. Math. France 30 (1902), 28-36.

5. X. Liu, Generalizations of de Montessus de Ballore's theorem on the row convergence of rational approximants, Ph.D. dissertation, University of South Florida, 1992.

6. X. Liu and E. B. Saff, Intermediate rows of the Walsh array of best rational approximant to meromorphic functions, Methods and Applications of Analysis, 2 (1995), 269-284.

7. E. B. Saff, Polynomials of interpolation and approximation to meromorphic functions, Trans. Amer. Math. Soc., 143 (1969), 509-522. 
8. __ An extension of Montessus de Ballore's theorem on the convergence of interpolating rational functions, J. Approx. Theory 6 (1972), 63-67.

9. J. Szabados, On some problems connected with polynomials orthogonal on the complex unit circle, Acta Math. Hungar., 33 (1979), 197-210.

10. H. Weyl, Gesammelte Abhandlungen, Vol. I, Springer-Verlag, New York, 1968.

Mathematics and Physics Department, University of La Verne, 1950 3rd Street, La Verne, CA 91750, USA 